\title{
Spatial filtering restricts the attentional window during both singleton and feature-based visual search
}

\author{
Nick Berggren ${ }^{1} \cdot$ Martin Eimer $^{1}$ \\ Published online: 28 January 2020 \\ (C) The Psychonomic Society, Inc. 2020
}

\begin{abstract}
We investigated whether spatial filtering can restrict attentional selectivity during visual search to a currently task-relevant attentional window. While effective filtering has been demonstrated during singleton search, feature-based attention is believed to operate spatially globally across the entire visual field. To test whether spatial filtering depends on search mode, we assessed its efficiency both during feature-guided search with colour-defined targets and during singleton search tasks. Search displays were preceded by spatial cues. Participants responded to target objects at cued/relevant locations, and ignored them when they appeared on the uncued/irrelevant side. In four experiments, electrophysiological markers of attentional selection and distractor suppression (N2pc and $\mathrm{P}_{\mathrm{D}}$ components) were measured for relevant and irrelevant target-matching objects. During singleton search, N2pc components were triggered by relevant target singletons, but were entirely absent for singletons on the irrelevant side, demonstrating effective spatial filtering. Critically, similar results were found for feature-based search. N2pcs to irrelevant target-colour objects were either absent or strongly attenuated (when these objects were salient), indicating that the feature-based guidance of visual search can be restricted to relevant locations. The presence of $\mathrm{P}_{\mathrm{D}}$ components to salient objects on the irrelevant side during feature-based and singleton search suggests that spatial filtering involves active distractor suppression. These results challenge the assumption that feature-based attentional guidance is always spatially global. They suggest instead that when advance information about target locations becomes available, effective spatial filtering processes are activated transiently not only in singleton search, but also during search for feature-defined targets.
\end{abstract}

Keywords Selective attention - Top-down control $\cdot$ Spatial filtering $\cdot$ Attentional capture $\cdot$ Event-related brain potentials . Feature-based attention

\section{Introduction}

To aid goal-directed behaviour in environments where multiple simultaneous visual objects and events compete with each

Significance statement Feature-based attention is a much-investigated topic in visual cognition. It is widely believed that feature-based attention operates in a spatially global fashion, and modulates visual processing independently of spatial attention. In the experiments reported here, we demonstrate that this is not true for feature-based attentional guidance in visual search. Using ERP markers of attentional selectivity, we show that feature-based attention is strongly modulated by spatial filtering, and that its effects are largely restricted to task-relevant locations in visual search displays. Our findings are important to correct common misperceptions of the nature of feature-based attention.

Nick Berggren

nbergg01@mail.bbk.ac.uk

1 Department of Psychological Sciences, Birkbeck College, University of London, Malet Street, London WC1E 7HX, UK other, selective attention is employed to prioritise current taskrelevant information. Attentional control processes can be space-based, facilitating the selection of objects at relevant locations, or feature-based, facilitating the processing of objects with distinct target-defining attributes (e.g. colour, shape, or size). Space-based attentional selection mechanisms are typically examined with spatial cueing procedures (e.g. Posner, 1980), where observers are given information about the location of upcoming target objects, while non-spatial attributes of these objects often remain unspecified. Featurebased attentional control is frequently studied using visual search tasks (e.g. Wolfe \& Horowitz, 2004), where target features are known in advance, but the location of target and distractor objects changes unpredictably across trials. During visual search, information about target-defining features is maintained as an attentional template or top-down task-set (Duncan \& Humphreys, 1992; Folk, Remington, \& Johnston, 1992), which can guide attentional selectivity towards objects with template-matching attributes. Because the 
location of search target objects is unknown in typical visual search tasks, feature-based attentional guidance has to operate in a spatially non-selective fashion, and prioritize templatematching features for all objects within a search display. As a result, these features will attract attention in a task-setcontingent fashion even if they belong to objects that have to be ignored (e.g. Eimer \& Kiss, 2008; Folk et al., 1992).

While space-based and feature-based attention are usually investigated separately, with different experimental paradigms, there are many situations where attentional selection could be guided jointly by both types of control processes. For example, an observer might search for their cell phone knowing that it will be somewhere on the floor but not on the desk or the shelf. In such cases, the optimal strategy would be to restrict the spatial scope of attentional guidance towards target-defining features by confining it to the relevant sections of visual space. The question whether such spatial filtering mechanisms can be activated during visual search when observers have advance knowledge about the location of target objects remains unresolved. There is clear evidence for the existence of spatial filtering in search tasks where one display item has an abrupt onset while all others appear gradually. When spatial attention is unfocused, the abrupt-onset item captures attention, and interferes with search performance when it is a distractor (Yantis \& Jonides, 1984). In contrast, when target location is indicated prior to the search display by valid spatial cues, abrupt-onset stimuli at other uncued locations have no effect on performance, indicating that attentional capture by abrupt onsets can be prevented by spatial filtering (Yantis \& Jonides, 1990; see also Ruthruff \& Gaspelin, 2018). Further evidence for effective spatial filtering comes from search tasks with feature singleton targets. Theeuwes and colleagues (e.g. Theeuwes, Kramer, \& Atchley, 2001) demonstrated that pre-cueing the hemifield where a singleton target defined by a colour change will appear strongly attenuates attentional capture by a salient distractor (another colour change) in the opposite hemifield. Reaction time (RT) costs produced by the presence of such distractors were significantly reduced on trials with spatial pre-cues, indicating that spatial filtering processes were activated. According to Theeuwes (2010), salient distractors are able to capture attention during singleton search only when they are presented within an attentional window that is under top-down endogenous control, but not when they appear outside this window (see also Belopolsky et al., 2007; Belopolsky and Theeuwes, 2010, Kerzel, Born, \& Schönhammer, 2012; Vatterott \& Vecera, 2015). On the other hand, it remains unclear whether such spatial filtering mechanisms can only be applied to prevent salience-driven attentional capture by irrelevant objects during search for feature singletons, or also in tasks where featurebased attentional guidance is required to distinguish target and distractor objects (see Bacon \& Egeth, 1994, for such a distinction between singleton and feature search modes). In fact, there is substantial evidence that feature-based attentional control operates in a spatially global fashion, both within and outside the current focus of spatial attention.

Evidence for the spatially global nature of feature-based attention was obtained in single-cell recording studies with nonhuman primates and in functional magnetic resonance imaging (fMRI) and event-related potential (ERP) studies with human participants (e.g. Martinez-Trujillo \& Treue, 2004; Saenz, Buracas, \& Boynton, 2002; Serences \& Boynton, 2007; Zhang \& Luck, 2009). Participants continuously monitored one of two dynamic bilateral stimulus displays in order to detect targets that were defined by one specific feature (e.g. a particular colour or movement direction). Even though spatial attention was fully focused in one hemifield, and stimuli in the other visual field could be entirely ignored, enhanced visual activations were found for target-matching as compared to nonmatching features in the irrelevant hemifield. These observations suggest that spatial filtering is unable to restrict the effects of feature-based attention to current task-relevant locations, even when attended and unattended object locations are widely separated in opposite hemifields. Similar results were obtained in behavioural and ERP experiments where observers monitored a central rapid serial visual presentation (RSVP) stream for feature-defined targets (e.g. Brignani, Lepsien, \& Nobre, 2010; Folk, Leber, \& Egeth, 2002; Leblanc, Prime, \& Jolicoeur, 2008; Leonard, Balestreri, \& Luck, 2015). Here, distractor objects with target-matching features attracted attention, in spite of the fact that they appeared at task-irrelevant lateral locations. It should be noted that these attentional capture effects tend to decrease with increasing distance from the currently attended location (e.g. Leonard et al., 2015; see also Stothart, Simons, Boot, \& Wright, 2019, for related observations), indicating that spatial filtering may modulate but not eliminate the effects of feature-based attention. Analogous observations come from experiments that measured steady-state visual evoked potentials (SSVEPs) to stimulus displays containing multiple stimuli flickering at different frequencies. When observers monitored feature-defined targets at one particular location, SSVEP amplitudes were enhanced for taskirrelevant stimuli with target-matching features in the to-beignored hemifield (Andersen, Fuchs, \& Müller, 2011; Andersen, Hillyard, \& Müller, 2013), again indicating that feature-based attention operates in a spatially global fashion.

The discrepancy between the evidence for strong spatial filtering during singleton search (e.g. Theeuwes et al., 2001) and the apparent lack of spatial filtering for feature-based attentional modulations of visual processing (e.g. Saenz et al., 2002) is striking. However, it is important to note that the spatially global nature of feature-based attention has primarily been demonstrated in experiments where observers continuously monitored streams of visual events within a fixed taskrelevant region of visual space. This is quite different from typical visual search tasks, where target and distractor objects 
appear at different unpredictable positions within search displays, and attention has to be rapidly allocated to new locations on successive trials. Thus, it remains an open question whether spatial filtering can restrict the spatial scope of feature-based attention during the selection of featuredefined targets in visual search tasks. One way to test this is to combine spatial cueing procedures with a standard visual search design. In such a hybrid cueing/search paradigm, spatial cues presented at the start of each trial signal to-beattended and to-be-ignored locations. These cues are followed by visual search displays that include feature-defined target objects, which require a response when they appear at a cued/relevant location, but have to be ignored when they are presented at uncued/irrelevant locations. If spatial filtering processes are activated during feature-based search, the ability of objects with target-matching features to attract attention should be strongly reduced when these are presented at uncued locations. This hypothesis was first investigated in a previous study from our lab (Seiss, Kiss, \& Eimer, 2009). Search displays contained one colour singleton object (e.g. a blue object among 11 uniformly grey distractors). Participants had to respond to this object when it had a particular target colour and appeared on the task-relevant side, which was indicated by a spatial cue. Objects in a different non-target colour and all objects on the uncued side had to be ignored. To assess the ability of target-colour objects to attract attention, $\mathrm{N} 2 \mathrm{pc}$ components were measured to these objects. The N2pc is an enhanced negativity elicited over posterior scalp electrodes contralateral to a candidate target object that appears among distractor objects. Typically emerging between 180 and $200 \mathrm{~ms}$ after search display onset, it is assumed to reflect the attentional selection of objects with task-relevant features during visual search (e.g. Eimer, 1996; Eimer \& Kiss, 2008; Luck \& Hillyard, 1994; Mazza, Turatto, Umiltà, \& Eimer, 2007). In the study by Seiss et al. (2009), reliable N2pc components were triggered by target-colour objects not only when these objects were presented at the cued/relevant side, but also when they appeared at the uncued/irrelevant side. Critically, these two N2pc components did not differ in amplitude, suggesting that there was little if any spatial filtering of targetmatching features at task-irrelevant locations.

These observations are in line with previous evidence that feature-based attention operates in a spatially global fashion, and suggest that this is also the case for typical feature-guided visual search tasks. However, objects with target-defining attributes were always feature singletons in the search task employed by Seiss et al. (2009). Although this task also included a go/no-go element based on the colour of these singletons, thereby encouraging a feature-based search strategy, the possibility remains that the $\mathrm{N} 2 \mathrm{pc}$ components to targetmatching objects reflect at least in part bottom-up attentional capture driven by the salience of these objects. In this context, it is important to note that when these objects were presented at uncued locations, N2pc components were followed by a contralateral positivity that started around $280 \mathrm{~ms}$ post-stimulus, and was entirely absent when the same objects appeared on the cued task-relevant side. Similar contralateral positivities have been observed in previous visual search studies in response to salient distractors (e.g. Sawaki \& Luck, 2010; Sawaki, Geng, \& Luck, 2012). This lateralised effect $\left(\mathrm{P}_{\mathrm{D}}\right.$ component; Hickey, Di Lollo, \& McDonald, 2009) is usually interpreted as a marker of active distractor inhibition processes (see Gaspelin \& Luck, 2018, for review). Across different studies, the latency of $\mathrm{P}_{\mathrm{D}}$ components varies considerably. Contralateral positivities have been observed prior to the N2pc time window (from 100 to $200 \mathrm{~ms}$ post-stimulus; e.g. Fortier-Gauthier et al., 2012), during this time window (200-300 ms post-stimulus; e.g. Hickey et al., 2009), or even later (300-400 ms poststimulus; e.g. Sawaki et al., 2012). This temporal variability could reflect different stages at which inhibitory mechanisms are activated. An early $P_{D}$ could reflect distractor inhibition acting to prevent any subsequent attentional capture, while a late $P_{D}$ may indicate an inhibition of distractor processing after an initial shift of attention to its location (Sawaki et al., 2012; see also Gaspelin \& Luck, 2018). The late $\mathrm{P}_{\mathrm{D}}$ observed in the Seiss et al. (2009) study may therefore suggest that although spatial filtering mechanisms are unable to prevent rapid salience-driven attentional capture by target-matching objects at irrelevant locations, they operate at a later stage, where this attentional bias is subject to active top-down inhibition. For these reasons, the results by Seiss et al. (2009) cannot be interpreted as unequivocal evidence for the spatially global nature of feature-based attentional guidance processes in visual search. Furthermore, recent behavioural results showed a reduction in the ability of distractor objects with target-matching features to attract attention when target locations were cued in advance (Burnham, 2018), suggesting that spatial filtering can modulate task-set contingent attentional capture. The present study was conducted to obtain more conclusive insights into the role of spatial filtering processes in both feature-based and singleton search tasks.

In all four experiments reported here, arrow cues presented at the start of each trial indicated the task-relevant location(s) in the subsequent search displays. Participants were instructed to find and report target objects at these relevant locations, and to ignore all objects on the opposite irrelevant side. To assess the effectiveness of spatial filtering, N2pc components were recorded to objects with target-matching attributes in these search displays, separately for trials where these objects appeared at a relevant location or in the irrelevant hemifield. If there was no spatial filtering at all, identical N2pc components should be observed on both types of trials. If spatial filtering was highly effective, it should prevent any allocation of attention to irrelevant objects, as indicated by the absence of N2pcs to targetmatching objects on the uncued side. A third possibility is that spatial filtering is partially effective, and this would be reflected 
by the presence of reliable but attenuated N2pc components on trials where these objects appear on the irrelevant side.

In Experiment 1, we compared feature-based and singleton search tasks. Arrow cues presented at the start of each trial indicated the to-be-attended task-relevant hemifield. In different blocks, targets were defined either by a single constant colour (One-Colour task) or by one of two equally probable colours (Two-Colour task). Participants were instructed to find and respond to target objects on the cued side (valid trials), and to ignore target-colour objects on the opposite uncued side (invalid trials). Valid and invalid trials were equiprobable and presented in random order. Search displays contained six objects, and the critical manipulation was whether the targetcolour object in these displays was a feature singleton. Homogeneous search displays contained one target-colour object among five grey distractors. In heterogeneous search displays, all six objects appeared in different colours. These two display types were presented in different blocks, resulting in four blocked task conditions (one-colour or two-colour search with homogeneous or heterogeneous search displays).

In blocks with homogeneous search displays, participants could adopt a colour-unspecific singleton search strategy, regardless of whether the target colour remained constant or changed across trials. Based on previous behavioural evidence that spatial filtering mechanisms are activated during singleton search (e.g. Theeuwes et al., 2001), we predicted that $\mathrm{N} 2$ pc components should only be elicited by colour singletons on the cued relevant side, but not by singletons on the uncued side. In blocks with heterogeneous search displays, attention had to be guided in a feature-based fashion. If feature-based attention is always spatially global, target-colour objects on the irrelevant side should elicit reliable N2pcs, and these may even be equal in size to the N2pcs triggered on valid trials, indicating that no spatial filtering was applied at all. Alternatively, N2pcs on invalid trials could be attenuated or absent, reflecting partially or fully effective spatial filtering during feature-based search. In addition, it is possible that the effectiveness of filtering also depends on how many colours (one vs. two) are represented by currently active colour target templates. In this case, differences may emerge between the One-Colour search task where the upcoming target colour was known in advance, and the Two-Colour task where the next target could appear in one of two possible colours.

\section{Experiment 1}

\section{Method}

\section{Participants}

Thirteen participants took part in Experiment 1. One participant was excluded from analysis due to a high number of EEG artifacts ( $>50 \%$ of all trials), leaving a final sample of $12(M=$ 28 years, $S D=7$; five male; two left-handed). All participants had normal or corrected-to-normal vision.

To determine a desired sample size, data from a previous study from our lab were utilised (Berggren \& Eimer, 2018), where search displays including colour-defined targets were preceded by target-colour probes at task-irrelevant locations. These probes elicited significant N2pc components, indicative of impaired spatial filtering $(d=1.50)$. Analysis using $G^{*}$ Power software suggested that replicating this effect required a minimum sample size of six participants assuming 0.8 power and an alpha level of .05 . We set our defined sample size at 12 participants, in line with previous N2pc investigations conducted in our and other labs (e.g. Berggren \& Eimer, 2018; Berggren, Jenkins, McCants, \& Eimer, 2017; Luck \& Hillyard, 1994).

\section{Stimuli and procedure}

The experiment was programmed and executed using E-Prime 2.0 software (Psychology Software Tools, Inc.). All stimuli were presented on a 24 -in. BenQ monitor $(60 \mathrm{~Hz} ; 1,920 \times$ 1,080 screen resolution) at a viewing distance of approximately $90 \mathrm{~cm}$, and the experiment was run on a SilverStone PC. Participants' responses were manually registered through a regular PC keyboard. All stimuli were presented on a black background, and a grey fixation $\operatorname{dot}\left(0.13^{\circ} \times 0.13^{\circ}\right.$ of visual angle) was present throughout each experimental block. Each trial contained an initial spatial cue display (100 ms duration) that was followed after a 700-ms blank interval by a search display appearing for $100 \mathrm{~ms}$ (see Fig. 1). The interval between the offset of a search display and the onset of the cue display on the next trial was 1,400 ms. Cue displays contained two left-pointing or right-pointing arrows appearing on either side of fixation $\left(0.32^{\circ} \times 0.32^{\circ}\right)$ at an eccentricity of $0.25^{\circ}$ from the fixation dot (measured relative to the edge of the arrows closest to fixation). Search displays contained six pentagonshaped objects presented with their point upward or downward $\left(1.40^{\circ} \times 1.40^{\circ}\right)$. They were located at the $45^{\circ}, 90^{\circ}$, $135^{\circ}, 225^{\circ}, 270^{\circ}$, and $315^{\circ}$ positions of a virtual circle at an eccentricity of $1.40^{\circ}$ from the fixation dot (measured relative to the edge nearest to fixation). In homogeneous search displays, one pentagon object appeared in colour while the remaining five objects appeared in grey. In heterogeneous search displays, all six objects appeared in a unique colour. The colours used were red (CIE colour coordinates: .605/ $.322)$, orange $(.543 / .409)$, yellow $(.405 / .470)$, green $(.296 /$ $.604)$, blue $(.169 / .152)$, magenta $(.270 / .134)$, or grey $(.305 /$ $.325)$. All colours were equiluminant $\left(14 \mathrm{~cd} / \mathrm{m}^{2}\right)$.

Participants' task was to direct their attention to the location indicated by the arrow cue presented at the start of each trial, in order to discriminate the shape of a colour-defined target object at that location. On half of all trials, the target appeared 


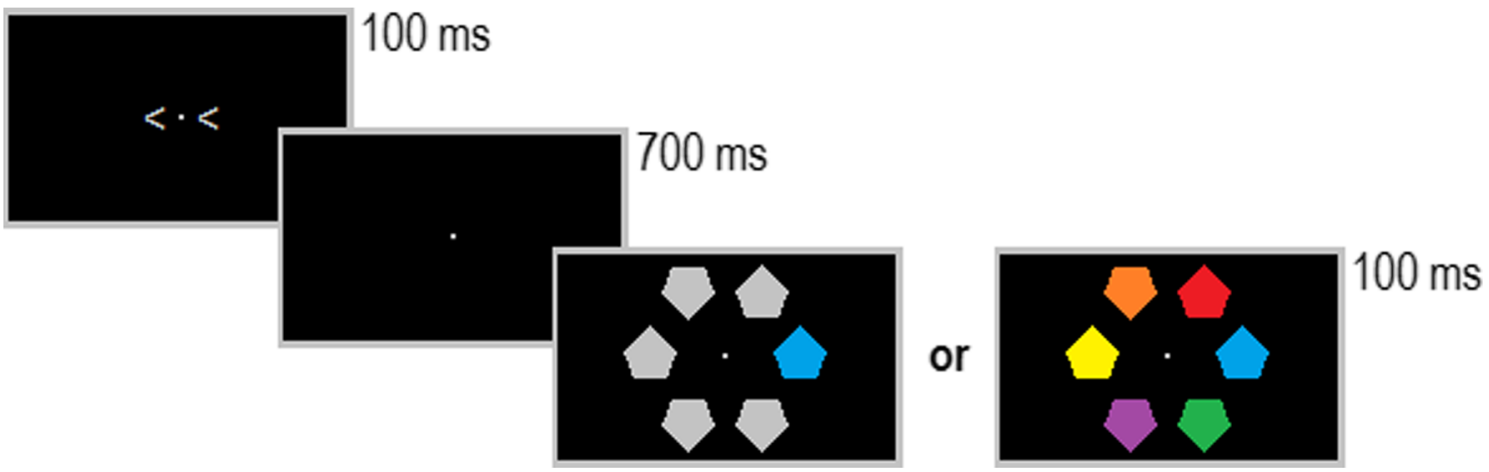

Fig. 1 Example experimental trial displays (not to scale) in Experiment 1. Each trial began with a left-pointing or right-pointing double arrow cue directing participants' attention to a task-relevant location in the left or right visual field. After a 700-ms interval, a search display appeared that contained a target-colour object (here: blue), either at the cued location (valid trials), or on the opposite uncued side (invalid trials). In homogeneous search displays, this object was the only coloured item. In heterogeneous search displays, it was accompanied by five distractors in five different colours. Participants had to report the shape of the targetcolour object on valid trials, and to refrain from responding on invalid trials where this object appeared on the uncued side

PO8, PO9, PO10, and Oz. A 500-Hz sampling rate with a 40$\mathrm{Hz}$ low-pass filter was used. Channels were referenced online to a left-earlobe electrode, and re-referenced offline to an average of both earlobes. No other filters were applied after EEG acquisition. Trials with eye blinks (exceeding $\pm 60 \mu \mathrm{V}$ at Fpz), horizontal eye movements (exceeding $\pm 30 \mu \mathrm{V}$ in the HEOG channels), and muscle movement artefacts (exceeding \pm $80 \mu \mathrm{V}$ at all other channels) were removed as artefacts, as were trials with response errors (including False Alarms on invalid trials). The remaining trials were segmented into epochs from $100 \mathrm{~ms}$ before to $500 \mathrm{~ms}$ after search display onset, relative to a 100-ms pre-stimulus baseline. Averaged ERP waveforms were computed for each task condition (One-Colour Homogeneous, One-Colour Heterogeneous, Two-Colour Homogeneous, Two-Colour Heterogeneous), separately for valid and invalid trials, and for trials with target-colour objects in the left and right visual field. N2pc amplitudes were quantified based on ERP mean amplitudes obtained at posterior electrode sites PO7 and PO8 between 200 and 300 ms after target display onset, in line with previous studies examining this component (e.g. Berggren et al., 2017; Berggren \& Eimer, 2018). We also investigated the possibility that inhibitionrelated $\mathrm{P}_{\mathrm{D}}$ components would be elicited on invalid trials, as in the previous study by Seiss et al. (2009). Because the $P_{D}$ emerged relatively late in this study (from about $280 \mathrm{~ms}$ poststimulus), we assessed $P_{D}$ mean amplitudes within a 280 - to 380 -ms post-stimulus time window. To investigate whether this component was already present earlier, we also checked for the presence of additional contralateral positivities on invalid trials between 100 and $200 \mathrm{~ms}$, and between 200 and 280 ms post-stimulus.

\section{Results}

Behavioural data Table 1 (top panel) shows RTs and error rates for valid trials, which were entered into a $2 \times 2$ 
Table 1 Reaction time (upper row) and error rate (lower row) data on trials with target-colour objects at cued/relevant locations in the OneColour and Two-Colour tasks of Experiments 1 and 2 (standard deviation in parentheses). Data are shown separately for homogeneous/ heterogeneous search displays (Experiment 1), and for diffuse/precise spatial cues (Experiment 2)

\begin{tabular}{lllll}
\hline Exp 1 & Homogeneous Displays & & \multicolumn{2}{l}{ Heterogeneous Displays } \\
& One-Colour & Two-Colour & One-Colour & Two-Colour \\
RTs $(\mathrm{msec})$ & $540(63)$ & $528(53)$ & $544(55)$ & $367(54)$ \\
Error $(\%)$ & $1(2)$ & $2(2)$ & Precise Spatial Cues & $3(5)$ \\
Exp 2 & Diffuse Spatial Cues & & One-Colour & Two-Colour \\
& One-Colour & Two-Colour & $547(77)$ & $578(93)$ \\
RTs $(\mathrm{msec})$ & $604(86)$ & $694(100)$ & $1(2)$ & $2(1)$ \\
Error $(\%)$ & $3(2)$ & $6(3)$ & & \\
\hline
\end{tabular}

repeated-measures analysis of variance (ANOVA) with the factors Display Type (Homogeneous, Heterogeneous) and Task (One-Colour Task, Two-Colour Task). A main effect of Display Type $\left(F(1,11)=7.43, p=.02, \eta_{\mathrm{p}}{ }^{2}=.40\right)$ reflected faster RTs in blocks with homogenous search displays relative to blocks with heterogeneous displays ( $M=534 \mathrm{vs}$. $555 \mathrm{~ms}$ ). There was no main effect of Task $(F<1)$, but a significant Display Type $\times$ Task interaction was present $(F(1,11)=6.52, p$ $\left.=.03, \eta_{\mathrm{p}}{ }^{2}=.37\right)$. Post hoc analysis showed that RTs did not differ between the One-Colour and Two-Colour tasks when search displays were homogeneous $(t(11)=1.09, p=.30)$. With heterogeneous displays, there was a trend for faster RTs in the One-Colour task than in the Two-Colour task (M $=544 \mathrm{vs} .567 \mathrm{~ms} ; t(11)=1.97, p=.075, d z=.57)$.

Error rates (incorrect or missed responses on valid trials and False Alarms on invalid trials) were analysed in a $2 \times 2$ $\times 2$ ANOVA, with the additional factor Validity. This analysis showed no main effect of Display Type $(F<1)$ or Task
$(F(1,11)=1.17, p=.30)$, but a significant main effect of Validity $\left(F(1,11)=6.08, p=.03, \eta_{\mathrm{p}}^{2}=.36\right)$, due to the fact that False Alarms on invalid trials were less frequent than response errors on valid trials $(M=1.58$ vs. $2.27 \%)$. There were no significant interactions for error rates.

N2pc data Figure 2 shows grand average ERP components elicited at electrodes PO7/8 contralateral and ipsilateral to the side of the target-colour objects on valid trials (top panels) and invalid trials (bottom panels), separately for the One-Colour and Two-Colour tasks, and blocks with homogeneous or heterogeneous search displays. Mean amplitudes obtained 200 $300 \mathrm{~ms}$ post-stimulus onset were entered into a $2 \times 2 \times 2 \times 2$ ANOVA with the factors Display Type (Homogeneous, Heterogeneous), Task (One-Colour Task, Two-Colour Task), Validity (Valid Trials, Invalid Trials), and Laterality (Ipsilateral, Contralateral). A significant main effect of Laterality $\left(F(1,11)=27.79, p<.001, \eta_{\mathrm{p}}{ }^{2}=.72\right)$ confirmed

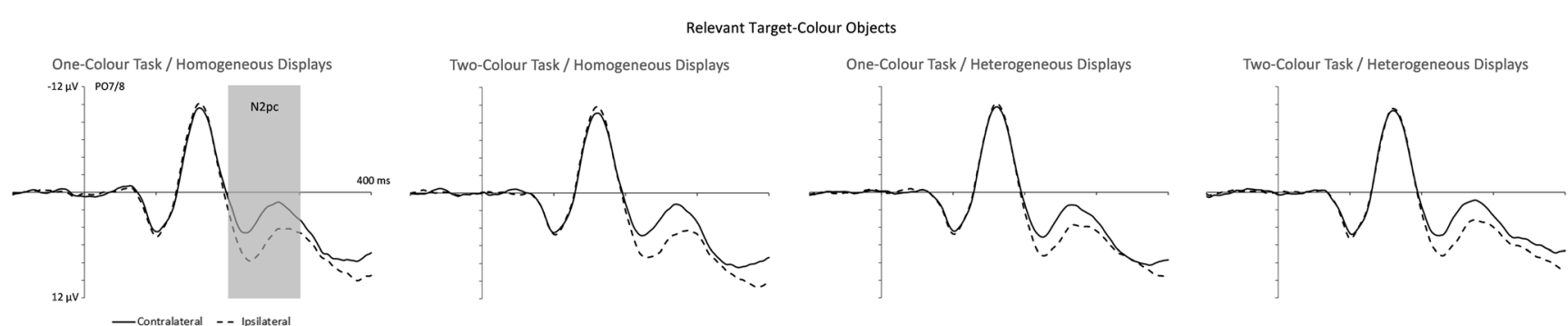

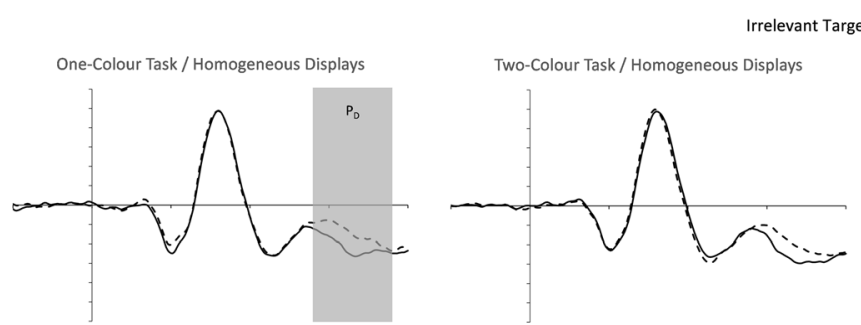

Fig. 2 Grand average event-related potential components (ERPs) obtained in Experiment 1 for search displays at electrode sites PO7/PO8 contralateral and ipsilateral to target-colour objects in the 400-ms interval after search display onset, relative to a $100-\mathrm{ms}$ pre-stimulus baseline. ERPs for valid trials where these objects appeared at cued/relevant

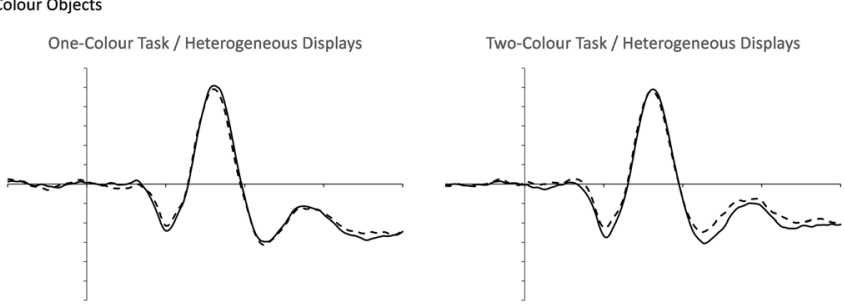

locations (top panels) and for invalid trials where they were presented uncued/irrelevant locations (bottom panels) are shown separately for blocks with homogeneous and heterogeneous search displays in the One-Colour and Two-Colour tasks 
the presence of reliable N2pc components. Critically, there was a significant Validity $\times$ Laterality interaction $(F(1,11)=$ $15.86, p<.005, \eta_{\mathrm{p}}{ }^{2}=.59$ ). Target-colour objects at taskrelevant cued locations elicited clear N2pc components $(M$ diff $=-2.39 \mu \mathrm{V} ; t(11)=5.57, p<.001, d z=1.61)$. In contrast, no reliable N2pc components were triggered when the same objects appeared at uncued irrelevant locations $(M \operatorname{diff}=.22$ $\mu \mathrm{V} ; t<1)$. In addition, there was a marginal trend for a fourway interaction (Laterality $\times$ Validity $\times$ Display Type $\times$ Task; $\left.F(1,11)=3.97, p=.07, \eta_{\mathrm{p}}{ }^{2}=.27\right)$.

To further assess the processing of target-colour objects at cued/relevant and at uncued/irrelevant locations, separate analyses were conducted for valid and invalid trials. Figure 3 shows N2pc difference waveforms obtained for valid trials by subtracting ERPs ipsilateral to the target from contralateral ERPs, separately for all four task conditions. N2pcs were clearly present in all conditions (contralateral vs. ipsilateral ERPs: $t$ 's $>5.23, p$ 's $<.001, d z$ 's $>1.52$ ), but N2pc amplitudes tended to be larger with homogeneous search displays. This was confirmed by a significant Display Type $\times$ Laterality interaction $\left(F(1,11)=5.27, p=.04, \eta_{\mathrm{p}}{ }^{2}=.32\right)$ for valid trials, demonstrating that $\mathrm{N} 2 \mathrm{pc}$ amplitudes were larger with homogeneous search displays $(M$ diff $=-2.69$ vs. -2.10 $\mu \mathrm{V})$. There were no other interactions involving the factor Laterality on valid trials $(F s<1)$. For invalid trials, Laterality did not interact with either Display Type $(F(1,11)$ $=1.43, p=.26)$ or Task $(F(1,11)=1.72, p=.22)$. Although there was a significant three-way interaction between these factors $\left(F(1,11)=5.66, p=.04, \eta_{\mathrm{p}}{ }^{2}=.34\right)$, follow-up analyses found no significant lateralisation in the N2pc time window for target-colour objects at irrelevant locations in homogeneous or heterogeneous search displays in either the OneColour or the Two-Colour tasks (all ts > 1.63, $p \mathrm{~s}>.13$ ).

As can be seen in Fig. 2 (bottom left panels), an enhanced contralateral positivity was triggered by target-colour objects

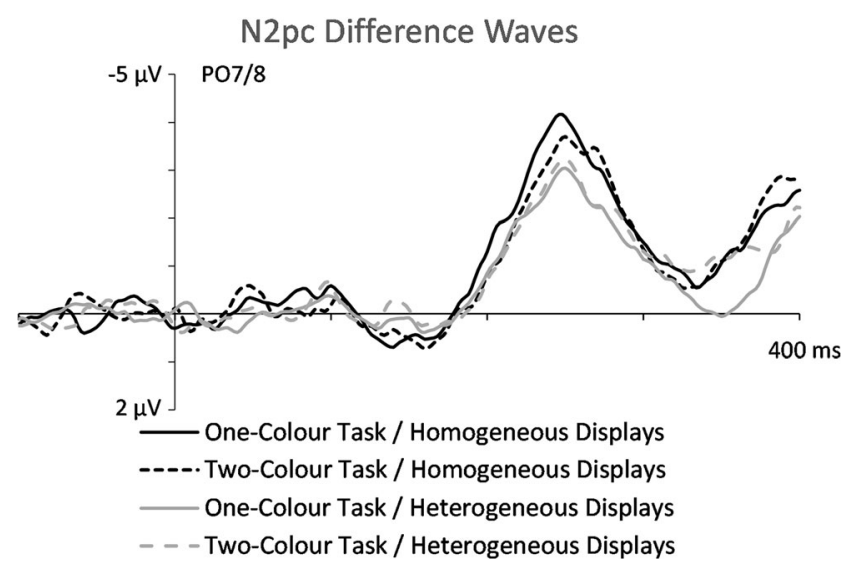

Fig. $3 \mathrm{~N} 2 \mathrm{pc}$ difference waveforms obtained by subtracting ipsilateral from contralateral event-related potential components (ERPs) elicited by target objects at cued/relevant locations in Experiment 1, shown separately for One-Colour and Two-Colour task blocks with homogeneous and heterogeneous search displays on the uncued side at around $280 \mathrm{~ms}$ post-stimulus, indicative of the suppression-related $P_{D}$ component. This positivity was much more pronounced in blocks with homogeneous search displays. An additional ANOVA was conducted for ERP mean amplitudes obtained on invalid trials within a 280 - to $380-\mathrm{ms}$ time window after search display onset, with the factors Display Type, Task and Laterality. This analysis obtained a significant main effect of Laterality $(F(1,11)=6.84, p=.02$, $\left.\eta_{\mathrm{p}}{ }^{2}=.38\right)$, as well as an interaction between Display Type and Laterality $\left(F(1,11)=5.05, p=.046, \eta_{\mathrm{p}}^{2}=.32\right)$. Follow-up analyses confirmed that a significant $\mathrm{P}_{\mathrm{D}}$ component was present only in blocks with homogeneous search displays ( $M$ diff $=1.28 \mu \mathrm{V} ; t(11)=3.12, p=.01, d z=.90)$, and not in blocks with heterogeneous search displays $(M \operatorname{diff}=.48 \mu \mathrm{V} ; t(11)=$ $1.37, p=.20$ ). There was no significant interaction between Task and Laterality nor any three-way interaction $(F \mathrm{~s}<1)$, indicating that irrelevant target-colour objects in homogeneous displays elicited $P_{D}$ components in both the OneColour and the Two-Colour tasks. No evidence for the presence of $\mathrm{P}_{\mathrm{D}}$ components was found during two earlier time windows (100-200 ms and 200-280 ms post-stimulus; main effects of Laterality: both $F_{\mathrm{s}}<1$ ).

\section{Discussion of Experiment 1}

The goal of Experiment 1 was to examine the role of spatial filtering in restricting the guidance of attention to task-relevant spatial locations during singleton and feature-based search. Participants did indeed appear to adopt different search strategies in blocks with homogeneous search displays and blocks with heterogeneous displays. RTs were fast during homogeneous search, and were unaffected by the predictability of the upcoming target colour (One-Colour vs. Two-Colour search) in these blocks. In blocks with heterogeneous search displays, RTs were generally slower. There was also a trend towards an additional RT cost when participants searched for two as compared to a single constant target colour, in line with previous evidence that attentional guidance is less efficient for multiple-colour as compared to single-colour search (e.g. Grubert \& Eimer, 2015). These behavioural results strongly suggest that a colour-unspecific singleton search mode was employed to find targets in homogeneous displays, whereas search was feature-based in blocks with heterogeneous displays. An alternative possibility that these two task conditions differed primarily in search difficulty (as targets could be detected more easily in homogeneous displays) rather than search strategies will be addressed in Experiment 3.

Critically, the ERP results provided strong evidence for spatial filtering not only during singleton search but also for feature search. Unsurprisingly, target objects at cued relevant locations generally elicited reliable N2pc components. In blocks with homogeneous search displays, no N2pc was triggered at all on invalid trials by target-colour objects on the 
irrelevant side. This is in line with previous behavioural studies demonstrating efficient spatial filtering during singleton search (e.g. Belopolsky et al., 2007, Belopolsky and Theeuwes, 2010; Theeuwes et al., 2001). Notably, a contralateral positivity ( $\mathrm{P}_{\mathrm{D}}$ component) was elicited in response to colour singletons between 280 and 380 ms post-stimulus onset, but only on trials where these objects appeared in the irrelevant visual field. As the $\mathrm{P}_{\mathrm{D}}$ has previously been associated with the top-down suppression of salient but taskirrelevant visual stimuli (e.g. Sawaki \& Luck, 2010), this result suggests that the processing of these stimuli outside the current attentional window might involve active inhibition. The most surprising result of Experiment 1 was the apparent presence of effective spatial filtering during feature-based search in heterogeneous search displays. No reliable N2pc components were triggered by target-colour objects on the uncued/irrelevant side, and this was the case in both the One-Colour and the Two-Colour search tasks. In contrast to singleton search, there was also no evidence for a subsequent $\mathrm{P}_{\mathrm{D}}$ component during feature-based search, suggesting that target-matching objects at irrelevant locations are subject to inhibition only when they are perceptually salient. This will be further assessed in Experiment 3.

The apparent efficiency of spatial filtering during featureguided visual search revealed by the $\mathrm{N} 2 \mathrm{pc}$ results of Experiment 1 contrasts markedly with prior evidence that feature-based attention operates in a spatially global fashion (e.g. Andersen et al., 2011, Martinez-Trujillo \& Treue, 2004; Serences \& Boynton, 2007; Seiss et al., 2009). For this reason, one goal of Experiment 2 was to replicate this result. Another goal was to examine whether the effectiveness of filtering during feature-based search is modulated by the size of the attentional window. In Experiment 1, spatial cues precisely indicated the location where validly cued target objects would appear (i.e., on the horizontal midline). In Experiment 2, an additional condition was included where cues indicated which hemifield was task-relevant, but validly cued target objects could appear at any of three locations within that hemifield. If spatial filtering was most efficient when the upcoming target position is known precisely, target-colour objects on the irrelevant side might be able to attract some attention in blocks with diffuse hemifield cues, as reflected by a reliable N2pc component to these objects.

\section{Experiment 2}

\section{Method}

\section{Participants}

Fourteen participants took part in Experiment 2. Two participants were excluded from analysis due to high artefact rejection rates ( $>50 \%$ of all trials). Of the remaining 12 participants, six were male and two were left-handed ( $M$ age $=31$ years; $\mathrm{SD}=7$ ). All had normal or corrected-to-normal vision.

\section{Stimuli and procedure}

These matched Experiment 1, with the following exceptions. Only heterogeneous search displays were shown. As in Experiment 1, participants searched either for a single constant target-colour object (One-Colour task) or for one of two possible target colours (Two-Colour task). Participants now completed 'precise' or 'diffuse' spatial cue blocks. In precise spatial cue blocks, arrow cues indicated the exact location where task-relevant target objects would be presented, as in Experiment 1. On invalid trials, target-colour objects always appeared at the exact opposite location. In diffuse spatial cue blocks, arrows only indicated the task-relevant hemifield, and target objects could appear at any of the three possible locations within this hemifield on valid trials. On invalid trials, target-colour objects could be presented at any of the three positions in the uncued hemifield.

The combination of cue type and task yielded four conditions (Diffuse One-Colour, Diffuse Two-Colour, Precise OneColour, Precise Two-Colour) that were each delivered in three successive experimental blocks of 96 trials each, with order of conditions randomised across participants.

\section{EEG recording and data analysis}

EEG recording and pre-processing was identical to Experiment 1. Averaged ERP waveforms were computed for each condition (One-Colour Diffuse, One-Colour Precise, Two-Colour Diffuse, Two-Colour Precise), separately for valid and invalid trials, and for trials with target-colour objects in the left and right visual field.

\section{Results}

Behavioural data Table 1 (bottom panel) shows reaction time and error rate data for valid trials in the four different task conditions. RTs on valid trials were analysed with a $2 \times 2$ ANOVA with the factors Cue Type (Precise Cue, Diffuse Cue) and Task (One-Colour Task, Two-Colour Task). A main effect of Cue Type $\left(F(1,11)=119.20, p<.001, \eta_{\mathrm{p}}{ }^{2}=.92\right)$ showed that RTs were faster with precise than with diffuse cues $(M=563$ vs. $649 \mathrm{~ms})$. There was also a main effect of Task $\left(F(1,11)=20.98, p=.001, \eta_{\mathrm{p}}{ }^{2}=.66\right)$, with faster RTs in One-Colour as compared to Two-Colour blocks ( $M=575$ vs. $636 \mathrm{~ms})$. In addition, a significant Cue Type $\times$ Task interaction was present $\left(F(1,11)=7.03, p=.02, \eta_{\mathrm{p}}^{2}=.39\right)$, as RTs were substantially slower in the Two-Colour task with diffuse cues than in the other three task conditions (see Table 1). Follow-up 
analyses showed that the RT benefits for precise versus diffuse cues were significant both in the One-Colour task $(t(11)=$ $3.80, p=.003, d z=1.10)$ and in the Two-Colour task $(t(11)$ $=9.44, p<.001, d z=2.73)$. RT benefits for the One-Colour versus Two-Colour task were present both with precise cues $(t(11)=3.09, p=.01, d z=.89)$ and with diffuse cues $(t(11)=$ $4.05, p=.002, d z=1.17)$.

Error rates (incorrect or missed responses on valid trials, False Alarms on invalid trials) were analysed with a $2 \times 2 \times 2$ ANOVA with the factors Cue Type, Task and Validity (Valid Trials, Invalid Trials). There was a significant main effect of Cue Type $\left(F(1,11)=33.86, p<.001, \eta_{\mathrm{p}}^{2}=.76\right)$, with more errors in blocks with diffuse spatial cues $(M=4$ vs. $2 \%)$, and a main effect of Task $\left(F(1,11)=8.42, p=.01, \eta_{\mathrm{p}}{ }^{2}=.43\right)$, with more errors in the Two-Colour task $(M=4$ vs. $2 \%)$. Cue Type and Task also interacted $\left(F(1,11)=9.53, p=.01, \eta_{\mathrm{p}}^{2}=.46\right)$. Although there were fewer errors with precise than with diffuse cues both in the One-Colour task $(t(11)=3.55, p=.005$, $d z=1.02)$ and in the Two-Colour search task $(t(11)=4.47, p=$ $.001, d z=1.29)$, this difference was larger in the Two-Colour task $(M$ diff $=5$ vs. $1 \%)$. There was no significant main effect of Validity or any other interactions $\left(F_{S}<1\right)$.

N2pc data Figure 4 shows grand average ERP components elicited following search display onset at electrodes PO7/8 contralateral and ipsilateral to the location of a target-colour object in the search display. These ERPs are shown separately for valid and invalid trials within One-Colour and Two-Colour tasks. Mean amplitudes obtained $200-300$ ms post-stimulus onset were entered into a $2 \times 2 \times 2 \times 2$ ANOVA with the factors Cue Type (Precise Cue, Diffuse Cue), Task (OneColour Target, Two-Colour Target), Validity (Valid Trials, Invalid Trials), and Laterality (Ipsilateral, Contralateral). This analysis found no interaction between Laterality and Cue Type $(F<1)$ and no higher-order interactions involving both factors (all $F<3.12, p>.10$ ), demonstrating that N2pc

\section{Relevant Target-Colour Objects}

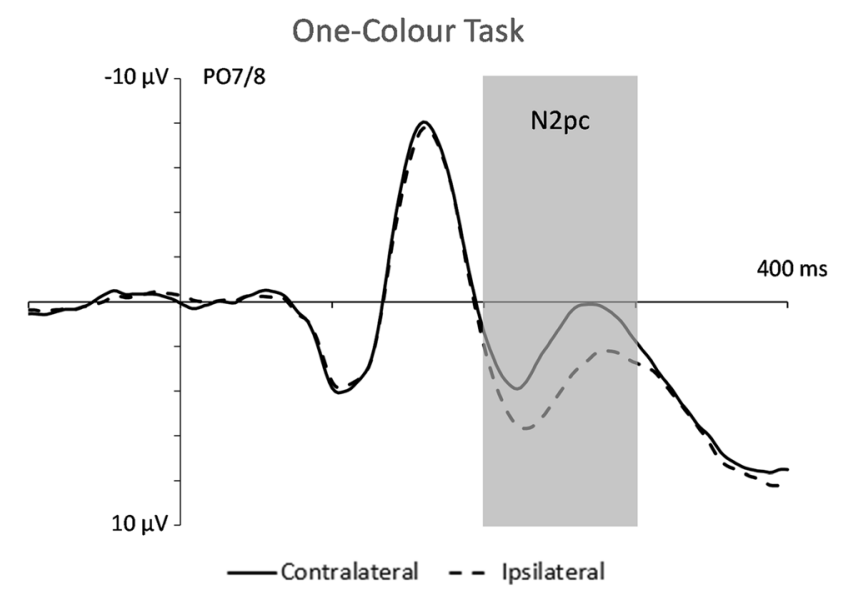

Two-Colour Task

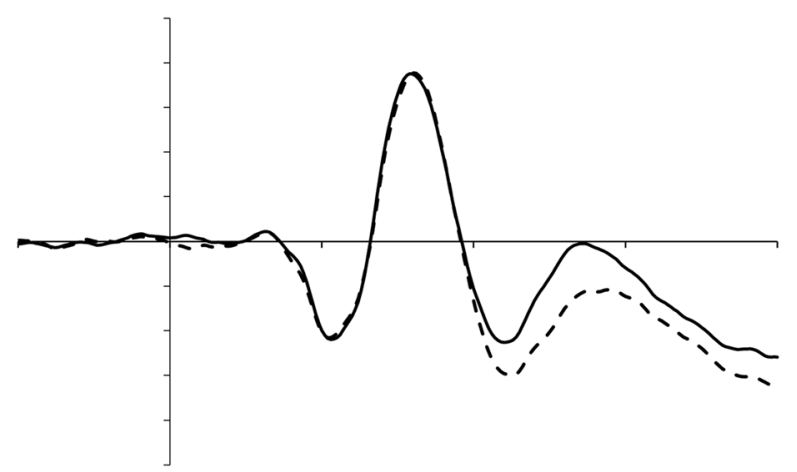

Irrelevant Target-Colour Objects

One-Colour Task

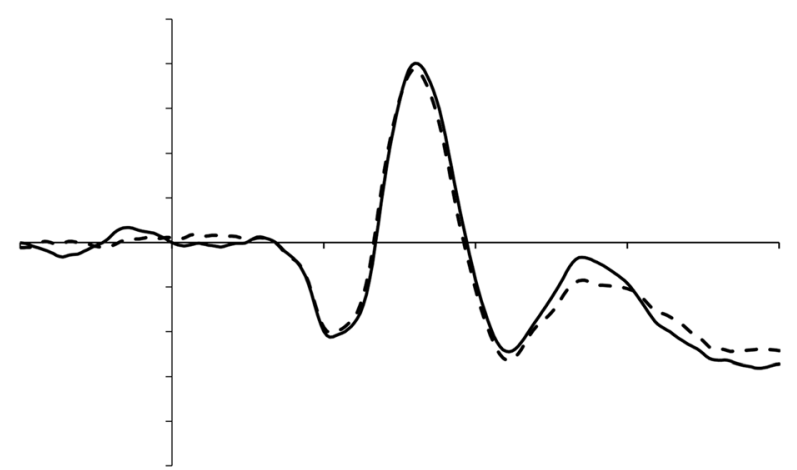

Fig. 4 Grand average event-related potential components (ERPs) obtained in Experiment 2 for search displays at electrode sites PO7/PO8 contralateral and ipsilateral to target-colour objects in the 400-ms interval after search display onset. ERPs are shown separately for trials where
Two-Colour Task

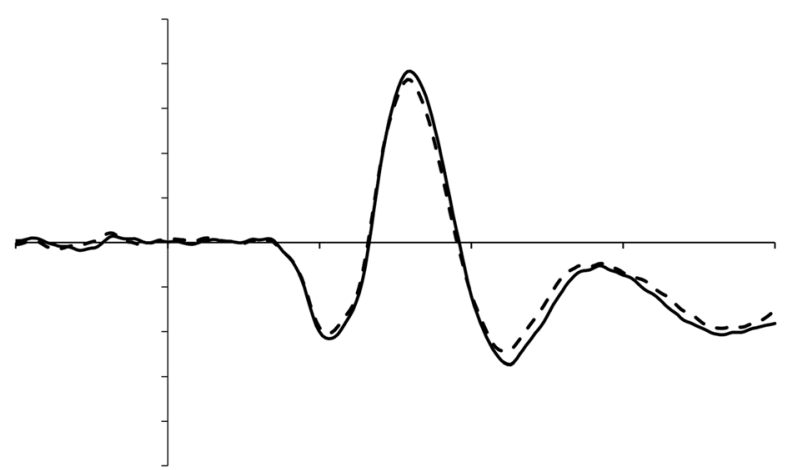

these objects appeared at cued/relevant and uncued/irrelevant locations (collapsed across trials with precise and diffuse cues), and for the OneColour and Two-Colour tasks 
amplitudes did not differ between blocks with precise and diffuse cues. For this reason, the ERP waveforms shown in Fig. 4 are collapsed across these two types of blocks.

A significant main effect of Laterality $(F(1,11)=56.18, p<$ $.001, \eta_{\mathrm{p}}{ }^{2}=.84$ ) demonstrated the presence of reliable N2pc components in Experiment 2. A significant Task $\times$ Laterality interaction was obtained $\left(F(1,11)=26.17, p<.001, \eta_{\mathrm{p}}{ }^{2}=.70\right)$, as $\mathrm{N} 2 \mathrm{pc}$ amplitudes were generally larger in the One-Colour task than in the Two-Colour task. Critically, there was a significant Validity $\times$ Laterality interaction $(F(1,11)=12.00, p=$ $\left..005, \eta_{\mathrm{p}}{ }^{2}=.52\right)$, reflecting systematic $\mathrm{N} 2 \mathrm{pc}$ differences between target-colour objects at cued versus uncued locations. Target-colour objects at task-relevant cued locations triggered clear N2pc components $(M$ diff $=-1.91 \mu \mathrm{V} ; t(11)=5.77, p<$ $.001, d z=1.67)$. In contrast, there was no overall reliable $\mathrm{N} 2 \mathrm{pc}$ to target-colour objects at uncued locations $(M$ diff $=$ $-.09 \mu \mathrm{V} ; t<1)$. A three-way interaction between Task, Validity and Laterality $\left(F(1,11)=6.11, p=.03, \eta_{\mathrm{p}}{ }^{2}=.36\right)$ was obtained. To assess this interaction, additional ANOVAs were conducted separately for valid and invalid trials, with the factors Task and Laterality (collapsed across blocks with precise and diffuse cues). On valid trials, there was no significant interaction between these two factors $(F(1,11)=1.98, p=.19)$. On invalid trials, this interaction was significant $(F(1,11)=19.36$, $\left.p=.001, \eta_{\mathrm{p}}{ }^{2}=.64\right)$. Target-colour objects at uncued taskirrelevant locations triggered a small but significant $\mathrm{N} 2 \mathrm{pc}$ component in the One-Colour task ( $M$ diff $=-.59 \mu \mathrm{V} ; t(11)$ $=2.53, p=.03, d z=.73)$. In the Two-Colour task, there was a tendency for a contralateral positivity in the $\mathrm{N} 2 \mathrm{pc}$ time range, but this was not reliable $(M$ diff $=.41 \mu \mathrm{V} ; t(11)=1.31, p=$ .22). Figure 4 (bottom left panel) also suggests that the small but reliable N2pc component to irrelevant target colour objects in the One-Colour task was followed by contralateral positivity. However, a mean amplitude assessment within a 280- to $380-\mathrm{ms}$ time-window did not find reliable evidence for the presence of a $\mathrm{P}_{\mathrm{D}}$ component $(M$ diff $=.25 \mu \mathrm{V} ; t(11)=$ $1.38, p=.20$ ).

\section{Discussion of Experiment 2}

The results of Experiment 2 provide further evidence that spatial filtering operates effectively during feature-based search. While target-colour objects at relevant locations elicited clear N2pc components, the same objects either failed to trigger any N2pc (in the Two-Colour task) or only a strongly attenuated N2pc (in the One-Colour task) when they appeared in the uncued visual hemifield. In addition, varying the precision of spatial cues and thus the size of the attentional window had no effect on spatial filtering. The fact that a small but reliable N2pc was observed for target-colour objects on the irrelevant side during One-Colour search suggests that when a single constant target colour template is active, templatematching objects at irrelevant locations might attract attention on at least some trials. However, since no reliable N2pc was found for these objects under identical conditions in Experiment 1, this conclusion has to remain tentative.

Overall, Experiment 2 confirmed the conclusion from Experiment 1 that feature-based attentional guidance in visual search can be restricted to currently task-relevant locations. This contrasts with previous N2pc results from our lab (Seiss et al., 2009), where N2pcs of similar size were found for target-colour objects at cued and at uncued locations. As noted earlier, these objects were feature singletons, and may thus have captured attention in a salience-based bottom-up fashion, even when they appeared on the irrelevant side. Because the search displays in the feature-based search tasks of Experiments 1 and 2 contained distractors in multiple different colours, irrelevant target-colour objects were no more salient than other distractors, thus eliminating the possibility of stimulus-driven attentional capture. The goal of Experiment 3 was to test whether spatial filtering in feature-based search is less efficient when target-colour objects are salient singletons. To assess this, we employed homogeneous search displays containing one coloured object among uniform grey items. As in Experiment 1, these displays were preceded by precise spatial cues that indicated the to-be-attended location on the left or right horizontal midline. To encourage a feature-based search mode, a go/no-go task was used where colour was relevant for response decisions. Participants were instructed to respond only to validly cued objects in one particular colour, and to withhold responses when an object in a different colour was presented at the cued location, as well to all coloured objects on the uncued side. A previous study from our lab (Eimer \& Kiss, 2010) has shown that this manipulation is sufficient to induce feature-based search, even when target objects are colour singletons. In this study, search displays were preceded by spatially informative colour singleton cues. In a task where observers searched for one of two possible colour singleton targets, both target-colour and non-targetcolour cues captured attention (as indicated by reliable behavioural spatial cueing effects), suggesting that a featureunspecific singleton search mode was active. Critically, in a second task where responses were required only to one of the two colour singletons, but had to be withheld when the other singleton appeared in a search display, only target-colour cues captured attention, demonstrating that search was now guided in a feature-based fashion. Employing an analogous go/no-go task in Experiment 3 should therefore also result in participants adopting a feature-specific search mode. If spatial filtering during feature-based search was less effective for salient singletons at irrelevant locations, these objects should trigger clear N2pc components in Experiment 3.

In Experiment 1, the comparison of spatial filtering efficiency during feature-based versus singleton search was complicated by the fact that these two tasks used different (heterogeneous vs. homogeneous) search displays. As the feature- 
based search task in Experiment 3 involved homogeneous search displays, it was now possible to directly contrast filtering between feature-based and singleton search with physically identical search displays. We therefore included an additional task condition in Experiment 3 where participants had to respond to any coloured object at the relevant location, regardless of its colour. Because search displays were identical in these two tasks, any differences in spatial filtering between them can thus be attributed to the currently active search mode. For example, if spatial filtering was more effective during singleton search, N2pc components to target-colour objects at irrelevant locations should be more strongly attenuated relative to feature-based search. Furthermore, if the inhibition-related $\mathrm{P}_{\mathrm{D}}$ component observed in Experiment 1 was specific to singleton search, it should not be found in the feature-based search task of Experiment 3 . This would also be consistent with previous findings by Barras and Kerzel (2016, 2017) indicating that distractor suppression, as reflected by $P_{D}$ components, is primarily elicited when search tasks are relatively easy. Alternatively, if salient items at irrelevant locations are actively inhibited regardless of search mode, a $P_{D}$ for invalidly cued target-colour objects should be present in both tasks.

\section{Experiment 3}

\section{Method}

\section{Participants}

Thirteen participants took part in Experiment 3. One participant was excluded from analysis due to a high number of artefacts (> 50\% of all trials), leaving a final sample of 12 ( $M=31$ years, $S D=7$; six male; one left-handed). All reported normal or corrected-to-normal vision.

\section{Stimuli and procedure}

These matched previous experiments with the following exceptions. Spatial cues indicated the precise location of targetcolour objects on valid trials on the horizontal midline, as in Experiment 1 and in the precise spatial cue condition of Experiment 2. Search displays always contained one coloured object (red or green) among grey distractors. Participants completed both a singleton and a feature-based search task (order counterbalanced between participants). In the Singleton task, they were instructed to respond to any coloured object (red or green) at the cued location, and to refrain from responding on invalid trials where a coloured object appeared on the uncued side. In the Feature task, they had to respond only when the coloured object at the cued location matched the target colour (red or green, counterbalanced across participants), and to withhold a response when this object appeared in the other 'no-go' colour, as well as on all invalid trials where a coloured object was presented on the uncued side. Following practice, participants completed 12 experimental blocks of 48 trials. There were four successive blocks for the Singleton task and eight successive blocks for the Feature task. This was done in order to match signal-to-noise ratios for N2pcs to target-colour objects between the two tasks, as only half of all trials in the feature task included a target-colour object. Task order was counterbalanced between participants.

\section{EEG recording and data analysis}

This was similar to previous experiments. Averaged ERP waveforms were computed for the Feature and Singleton tasks, separately for valid and invalid trials and trials with coloured objects in the left and right visual field. For the Feature task, ERPs were obtained separately for search displays where the coloured object matched the go or no-go colour.

\section{Results}

Behavioural data RTs to target objects at cued locations were faster in the Singleton task relative to the Feature task $(M=$ 519 vs. $551 \mathrm{~ms} ; t(11)=3.91, p=.002, d z=1.13)$. Error rates were generally very low $(M=1 \%)$, and did not differ between the two tasks $(t<1)$.

N2pc data Figure 5 shows grand average ERP components elicited at electrodes PO7/PO8 contralateral and ipsilateral to the side of the colour objects on valid trials and invalid trials, shown separately for the Singleton and Feature tasks. A $2 \times 2$ $\times 2$ ANOVA was conducted within the N2pc time window used in the first two experiments (200-300 ms post-stimulus), with the factors Task (Singleton, Feature), Validity (Valid Trials, Invalid Trials) and Laterality (Ipsilateral, Contralateral). For the Feature task, only ERPs in response to search displays with a go-colour object were included. This showed a significant main effect of Laterality $(F(1,11)$ $\left.=13.80, p=.003, \eta_{\mathrm{p}}{ }^{2}=.56\right)$, no interaction between Laterality and Task $(F(1,11)=2.23, p=.16)$, but a significant interaction between Laterality and Validity $\left(F(1,11)=29.65, p<.001, \eta_{\mathrm{p}}{ }^{2}\right.$ $=.73)$. A reliable $\mathrm{N} 2 \mathrm{pc}$ component was present for coloured target objects at cued/relevant locations $(M \operatorname{diff}=-2.57 \mu \mathrm{V}$; $t(11)=5.23, p<.001, d z=1.51)$, but not when objects with the target colour appeared on the irrelevant side $(M$ diff $=.09$ $\mu \mathrm{V} ; t<1)$. There was no significant three-way interaction $(F(1,11)=2.03, p=.18)$.

As seen in Fig. 5 (bottom right panel), there appears to be an N2pc to target-colour object at irrelevant locations in the Feature task, but this component emerged slightly earlier than in the feature-based search tasks in Experiments 1 and 2. This 
Relevant Target-Colour Objects

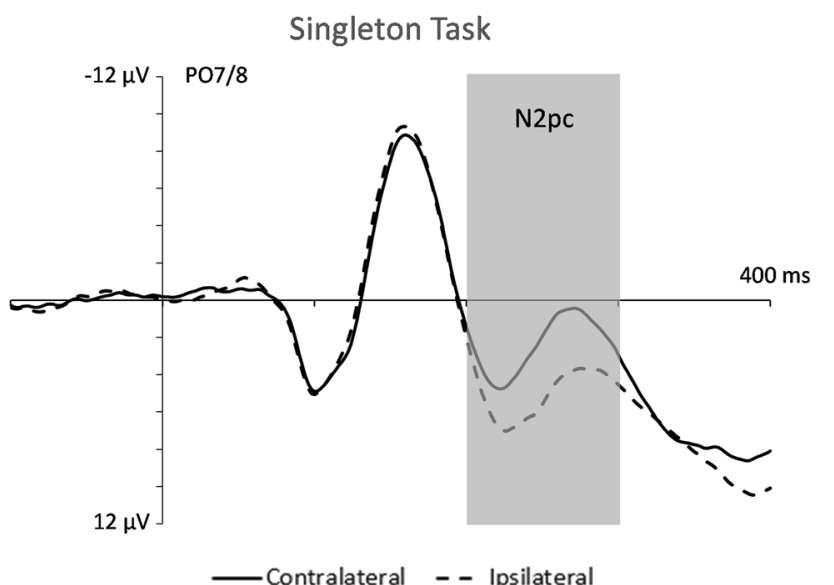

Feature Task

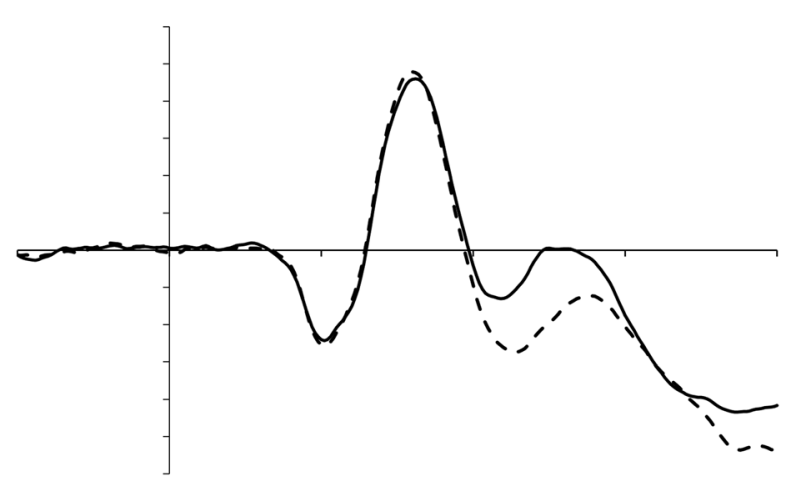

Irrelevant Target-Colour Objects

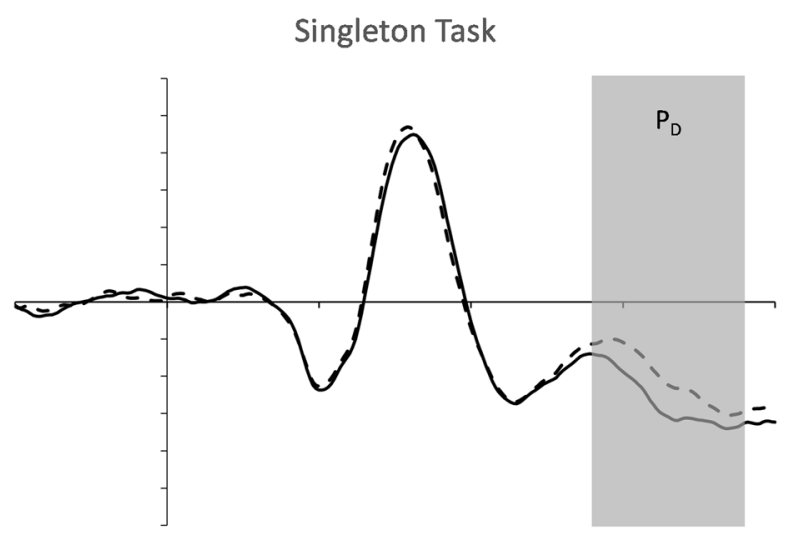

Fig. 5 Grand average event-related potential components (ERPs) obtained in Experiment 3 for search displays at electrode sites PO7/PO8 contralateral and ipsilateral to target-colour objects in the 400-ms interval

is likely due to the fact that heterogeneous search displays without singletons were used in Experiments 1 and 2, whereas target-matching objects were salient feature singletons in Experiment 3. In addition, this N2pc was followed by a contralateral positivity $\left(\mathrm{P}_{\mathrm{D}}\right.$ component) starting at around $270 \mathrm{~ms}$ post-stimulus, which was also evident for irrelevant coloured objects in the Singleton task (bottom left panel). The presence of this $\mathrm{P}_{\mathrm{D}}$ component might have been responsible for the absence of a reliable N2pc for irrelevant coloured objects in the Feature task within our standard 200- to 300 -ms poststimulus N2pc measurement window. To assess this, an earlier 180- to 280-ms post-stimulus time window was applied post hoc. Within this window, a reliable N2pc was indeed present on invalid trials in the Feature task $(M$ diff $=-.70 \mu \mathrm{V} ; t(11)=$ $2.43, p=.03, d z=.70$ ), whereas no evidence for an $\mathrm{N} 2 \mathrm{pc}$ was found for invalid trials in the Singleton task $(M \operatorname{diff}=-.01 \mu \mathrm{V}$; $t<1$ ). The difference in N2pc mean amplitudes between these two tasks was also significant $(t(11)=2.72, p=.02, d z=.79)$.

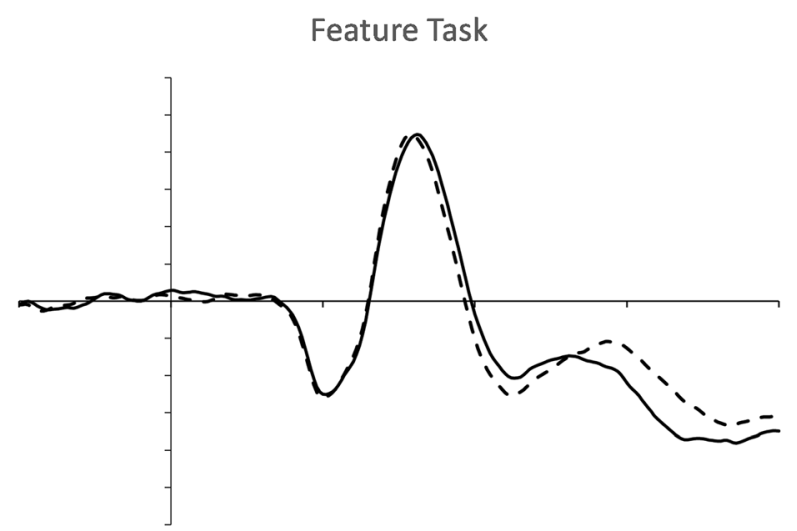

after search display onset. ERPs are shown separately for trials where these objects appeared at cued/relevant and uncued/irrelevant locations, and for the Singleton and Feature tasks

The contralateral positivity indicative of the $\mathrm{P}_{\mathrm{D}}$ component observed on invalid trials in both tasks was assessed on the basis of mean amplitudes measured within a 280 - to 380-ms post-stimulus onset time window. A $2 \times 2$ ANOVA with the factors Task and Laterality showed a main effect of Laterality $\left(M\right.$ diff $\left.=1.45 \mu \mathrm{V} ; F(1,11)=11.75, p=.006, \eta_{\mathrm{p}}{ }^{2}=.52\right)$, but no interaction between Laterality and Task $(F(1,11)=1.29, p=$ .28 ), indicating that $\mathrm{P}_{\mathrm{D}}$ components were present in both tasks.

\section{Discussion of Experiment 3}

Experiment 3 investigated the efficiency of spatial filtering during feature-guided search under conditions where targetmatching objects at irrelevant locations were salient singletons. In line with the results of Experiments 1 and 2, clear evidence for spatial filtering was found in the Feature task of Experiment 3, as N2pcs to target-colour objects were strongly 
attenuated when these objects appeared on the irrelevant side. The N2pc for irrelevant target-colour objects in this task was not reliable in the main analysis, but was significant when a slightly earlier time window (180-280 ms post-stimulus) was employed post hoc. While this observation might suggest that spatial filtering did not completely prevent the allocation of attention to these objects, the main conclusion from the N2pc results in the Feature task is that spatial filtering remains effective during feature-based search even when irrelevant target-matching objects are physically salient.

The results of the Singleton task were essentially identical to those observed for the corresponding task in Experiment 1. N2pcs were only triggered by target objects on the relevant side but not by colour singletons on the irrelevant side, indicating that spatial filtering was highly effective in preventing any attentional capture by irrelevant singletons. Because the search displays used in these two tasks were physically identical, the presence of a reliable N2pc to target-colour objects on the uncued side in the Feature task and the absence of an N2pc to these objects in the Singleton task suggests that spatial filtering may be generally more efficient during singleton search. Also in line with Experiment 1, a $\mathrm{P}_{\mathrm{D}}$ component was elicited by colour singletons on the irrelevant side in the Singleton task, indicative of active inhibition. Importantly, this $\mathrm{P}_{\mathrm{D}}$ component was also present in the Feature task. This result indicates that the suppression of salient feature singletons on the irrelevant side is not specific to singleton search, but occurs whenever a physically salient object is encountered at a task-irrelevant location, also during feature-based search. The absence of $\mathrm{P}_{\mathrm{D}}$ components to irrelevant target-colour objects in the heterogeneous search displays in Experiments 1 and 2 suggests that a match with a currently active colour template is not sufficient for such suppressive mechanisms to be triggered, and that bottom-up salience is a necessary condition for distractor inhibition.

The presence of strong spatial filtering during featurebased search demonstrated again in Experiment 3 remains inconsistent with the results of a previous study that used similar procedures (Seiss et al., 2009), but found N2pc evidence for ineffective filtering.

One notable difference from the current Experiment 3 was that neutral cues were presented on one-third of all trials in this earlier study. These cues instructed participants to not apply any spatial filtering, but to respond to all target-colour objects, regardless of their location. The inclusion of trials with neutral cues may have generally discouraged participants from spatial filtering, also on trials where informative cues were presented. We tested this possibility in Experiment 4. Here, one blocked task condition was identical to the feature task of Experiment 3 , and included no neutral cues. In other blocks, neutral arrow cues pointed in both directions on a randomly intermixed third of all trials. These neutral cues indicated that no spatial filtering was required, as participants had to respond to all go- colour objects, regardless of whether they appeared on the left or right display side. If spatial filtering is less effective when such neutral cue trials are included, N2pc components to target-colour objects at irrelevant locations should be larger relative to blocks without neutral cues.

The fact that N2pc components to target-colour objects were found to emerge slightly earlier in Experiment 3 is likely due to the fact that these objects were salient colour singletons in this experiment, but not in Experiments 1 and 2. Several previous studies have shown that N2pcs tend to be triggered more rapidly in response to singleton targets as compared to less salient feature-defined targets (e.g. Callahan-Flintoft \& Wyble, 2017; FeldmannWüstefeld \& Schubö, 2015; Mazza, Turatto, \& Caramazza, 2009). Because Experiment 4 again employed target-colour singleton objects, the time window for N2pc amplitude analyses was adjusted accordingly.

\section{Experiment 4}

\section{Method}

\section{Participants}

Twelve participants took part in Experiment $4(\mathrm{M}$ age $=33$ years, $\mathrm{SD}=7$; four male; all right-handed). All participants reported normal or corrected-to-normal vision.

\section{Stimuli, procedure and data analyses}

These matched the Feature task of Experiment 3, with the following exceptions. In blocks with no neutral cues, procedures were identical to the Feature task of Experiment 3. Responses were required when the colour singleton at the cued location matched the target colour, and had to be withheld when this singleton matched the other 'no-go' colour. In the new condition where blocks contained neutral cues, arrow cues pointed in both directions on one-third of all trials. Participants had to respond to a go-colour target object regardless of whether it appeared in the left or right visual field on these trials. Following practice, participants completed $20 \mathrm{ex}-$ perimental blocks of 48 trials. Twelve of these blocks contained neutral cue trials, and the remaining eight blocks contained no neutral cue trials. All blocks with neutral cues and all blocks without such cues were presented successively, with block order counterbalanced between participants. Based on the N2pc results of Experiment 3, which used identical search displays including colour singletons, N2pc mean amplitudes were computed within a 180 - to 280 -ms post-stimulus time window. 


\section{Results}

Behavioural data RTs to cued target-colour objects on trials where cues were informative (i.e., pointed to the left or right side) did not differ between the blocks with and without neutral cues $(M=578$ vs. $577 \mathrm{~ms} ; t<1)$. In blocks with neutral cues, RTs were delayed on trials where a neutral cue was presented relative to trials with informative cues $(M=615$ vs. $578 \mathrm{~ms} ; t(11)=4.29, p=.001, d z=1.24)$. For error rates, there was a trend for more errors in blocks with neutral cues $(M=2$ vs. $1 \% ; t(11)=1.86, p=.09, d z=.54)$. This was primarily due to the fact that False Alarms on trials where a go-colour object appeared on the irrelevant side were more frequent in these blocks relative to blocks without neutral cues $(M=5$ vs. $2 \% ; t(11)=2.52, p=.03, d z=.73)$.

N2pc data Figure 6 shows grand average ERP components elicited at PO7/PO8 contralateral and ipsilateral to the side of target-colour objects on valid trials and invalid trials, shown separately for blocks with and without neutral cues. Mean amplitudes obtained $180-280 \mathrm{~ms}$ post-stimulus onset were entered into a $2 \times 2 \times 2$ ANOVA with the factors Block Type (No Neutral cues, Neutral cues), Validity (Valid, Invalid) and Laterality. For blocks with neutral cues, trials with neutral cues were excluded, to ensure that identical types of trials were analysed in both task conditions. A significant main effect of Laterality $\left(F(1,11)=36.22, p<.001, \eta_{\mathrm{p}}{ }^{2}=.77\right)$ indicated the reliable presence of N2pc components. There was no Block Type $\times$ Laterality interaction $(F<1)$, but a significant Validity $\times$ Laterality interaction $(F(1,11)=9.58$, $\left.p=.01, \eta_{\mathrm{p}}^{2}=.47\right)$ was present. As in the three previous experiments, larger $\mathrm{N} 2 \mathrm{pc}$ components were elicited on valid trials $(M$ diff $=-2.75 \mu \mathrm{V} ; t(11)=7.46, p<.001, d z=2.15)$ relative to invalid trials. Importantly, reliable N2pc components were also present on invalid trials where go-colour objects appeared on the irrelevant side $(M$ diff $=-1.40 \mu \mathrm{V} ; t(11)$

\section{Relevant Target-Colour Objects}

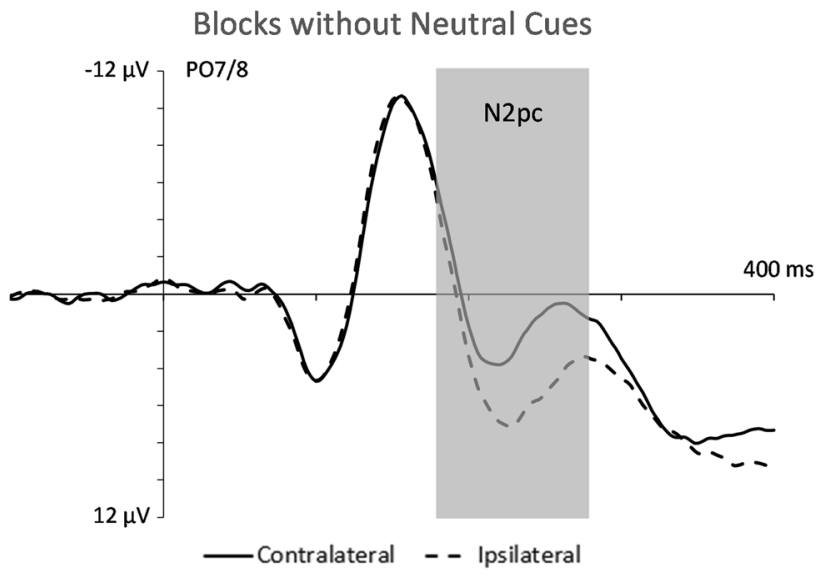

Blocks with Neutral Cues

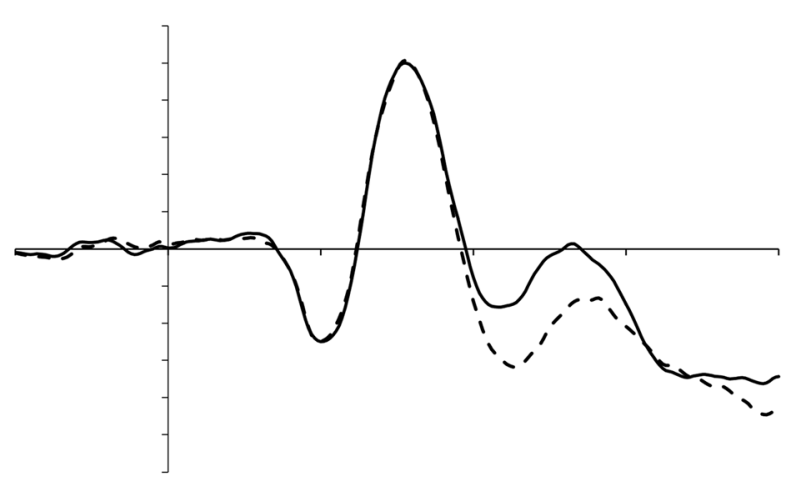

Irrelevant Target-Colour Objects

Blocks without Neutral Cues

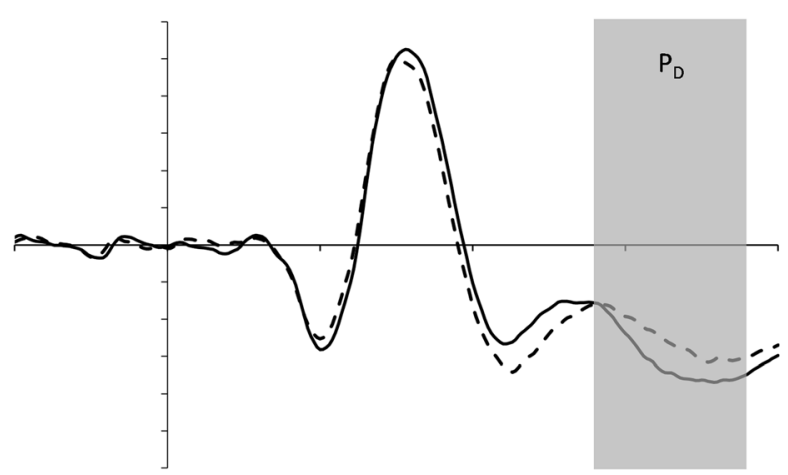

Fig. 6 Grand average event-related potential components (ERPs) obtained in Experiment 4 for search displays at electrode sites PO7/PO8 contralateral and ipsilateral to target-colour objects in the 400-ms interval after search display onset. ERPs are shown for trials where these objects
Blocks with Neutral Cues

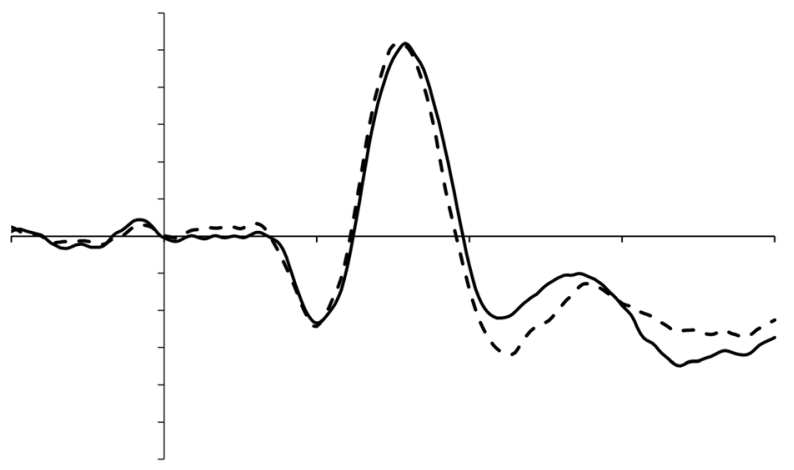

appeared at cued/relevant and uncued/irrelevant locations, separately for blocks that included no-filtering trials with neutral cues, and blocks without neutral cue trials 
$=3.14, p=.009, d z=.91)$. Paired t-tests comparing ipsilateral versus contralateral mean amplitudes confirmed that reliable $\mathrm{N} 2 \mathrm{pc}$ components were present for valid as well as invalid trials, in blocks both with or without neutral cues $(t$ 's $>2.50$, $p \mathrm{~s}<.03, d z \mathrm{~s}>.72$ ). Importantly, there was also a significant three-way interaction between Validity, Laterality, and Block Type $\left(F(1,11)=5.30, p=.04, \eta_{\mathrm{p}}{ }^{2}=.33\right)$. This was due to the fact that spatial filtering effects (i.e., the attenuation of $\mathrm{N} 2 \mathrm{pc}$ amplitudes on invalid as compared to valid trials) were more pronounced in blocks without neutral cues $(M$ diff $=-1.67 \mu \mathrm{V}$; $t(11)=3.06, p=.01, d z=.88)$ relative to blocks where neutral cues were included $(M \operatorname{diff}=-1.04 \mu \mathrm{V} ; t(11)=2.96, p=.01$, $d z=.85$ ).

Finally, as in previous experiments, $\mathrm{P}_{\mathrm{D}}$ components were triggered by colour singleton objects on the irrelevant side (Fig. 6, bottom panels). A $2 \times 2$ ANOVA was conducted for mean amplitudes obtained within a 280 - to 380 -ms post-stimulus time window on invalid trials, with the factors Block Type and Laterality. A significant main effect of Laterality $\left(F(1,11)=5.74, p=.04, \eta_{\mathrm{p}}{ }^{2}=.34\right)$ was present, which did not interact with Block Type $(F<1)$, indicating that reliable $\mathrm{P}_{\mathrm{D}}$ components $(M \operatorname{diff}=1.06 \mu \mathrm{V})$ were equally present in both tasks.

\section{Discussion of Experiment 4}

Experiment 4 again demonstrated clear spatial filtering effects during feature-based visual search, as reflected by significantly attenuated N2pc components for target-colour objects at irrelevant locations. However, although the N2pcs elicited by these objects were small, they were still reliably present, indicating that spatial filtering did not completely prevent attentional capture. As in all previous experiments where targetcolour objects were feature singletons, reliable $\mathrm{P}_{\mathrm{D}}$ components indicative of active suppression were triggered when these objects were presented at irrelevant locations. The main goal of Experiment 4 was to test whether the inclusion of trials with neutral cues where no spatial filtering was required would reduce the effectiveness of spatial filtering. Evidence for this was indeed found. The effects of spatial filtering on the $\mathrm{N} 2 \mathrm{pc}$ (i.e., the reduction of $\mathrm{N} 2 \mathrm{pc}$ amplitudes to target-colour objects on the irrelevant side relative to targets at the cued location) were reliably smaller in blocks where neutral cues appeared on one-third of all trials relative to blocks without neutral cues. This was also reflected in performance, as False Alarms to target-colour objects on the to-be-ignored side were more frequent in blocks that included neutral trials. This reduction in the efficiency of spatial filtering observed in Experiment 4 as a result of including no-filtering trials partially reconciles the findings of the present study with the results of a previous N2pc experiment that also included neutral cues (Seiss et al., 2009), where little evidence for spatial filtering was obtained. However, even in the task including neutral cues in Experiment 4, N2pcs were still significantly attenuated for irrelevant as compared to relevant target-colour objects, demonstrating that spatial filtering processes remained active. More generally, the fact that clear-cut N2pc evidence for effective spatial filtering during feature-guided visual search was obtained in all four experiments of the present study strongly suggests that the results of this earlier study (Seiss et al., 2009) have to be regarded as an exception to this general rule. Filtering processes may be relatively inefficient under specific conditions, such as when filtering and no-filtering trials are intermixed, and objects at task-irrelevant locations are highly salient. In the latter case, an additional important factor is display set size, as singleton objects become more salient, and can be detected more rapidly, when the number of homogenous items in the search display is increased (e.g. Bacon \& Egeth, 1991; Bravo \& Nakayama, 1992). For this reason, the larger display set size of 12 objects employed by Seiss et al. (2009) may have further increased the salience of irrelevant target-colour singletons, resulting in strong bottomup attentional capture counteracting the effects of spatial filtering at the level of N2pc components. Overall, the balance of the currently available evidence strongly suggests that spatial filtering is highly effective during feature-based visual search. The implications of this conclusion in the context of previous work on feature-based attention are considered in detail below.

\section{General discussion}

The goal of the current study was to examine the role of spatial filtering in restricting the allocation of attention during visual search. Previous research has suggested that spatial filtering is highly efficient during singleton search, where it can prevent the capture of attention by salient objects outside the current attentional window (e.g. Belopolsky et al., 2007, Belopolsky and Theeuwes, 2010; Theeuwes et al., 2001). In contrast, it is generally assumed that feature-based attention operates in a spatially global fashion, and enhances the processing of objects with target-matching features both within and outside the focus of spatial attention (e.g. Andersen et al., 2011; Folk et al., 2002; Leblanc et al., 2008; Martinez-Trujillo \& Treue, 2004; Saenz et al., 2002; Seiss et al., 2009; Serences \& Boynton, 2007). If this was correct, spatial filtering mechanisms should only be effective during visual search for feature singletons, but not in search tasks where attentional guidance is feature-based. Here, we investigated this hypothesis in four experiments where N2pc components were measured as markers of allocating attention in response to target-colour objects that appeared either at relevant locations or in the opposite irrelevant visual field. Strong evidence of spatial filtering was found not only in singleton search tasks, but also consistently in tasks where participants had to adopt a featurebased search mode. In both types of tasks, large N2pc 
components were observed for target-colour objects at relevant locations, but these components were strongly attenuated or entirely absent when the same objects appeared in the irrelevant hemifield. Thus, the general conclusion is that spatial filtering mechanisms can be effectively applied both during singleton search as well as in feature-based search tasks.

In the singleton search tasks of Experiments 1 and 3, colour singletons presented among grey distractors triggered $\mathrm{N} 2 \mathrm{pc}$ components only when they were presented at cued/relevant locations, but not when they appeared in the uncued irrelevant hemifield. This is entirely in line with the hypothesis that salient distractors will not capture attention during singleton search when these distractors are located outside the current window of spatial attention (e.g. Theeuwes, 2010). The current results also suggest that the prevention of attentional capture by irrelevant singletons involves active top-down inhibition. Salient singletons in the irrelevant hemifield consistently elicited a contralateral positivity from approximately $280-380 \mathrm{~ms}$ post-stimulus onset, which is likely to reflect the inhibitionrelated $\mathrm{P}_{\mathrm{D}}$ component observed in many previous studies (e.g. Sawaki \& Luck, 2010; Sawaki, Geng, \& Luck, 2012). According to Sawaki and Luck (2010), salient distractor stimuli elicit an 'attend-to-me' signal during visual search, which can result either in attentional capture (reflected by an N2pc) and/or in active suppression (reflected by the $\mathrm{P}_{\mathrm{D}}$ ). Notably, $\mathrm{P}_{\mathrm{D}}$ components to irrelevant singletons were observed in the present study not only during singleton search, but also in featurebased search tasks (Experiments 3 and 4), suggesting that active inhibition is generally triggered by salient but taskirrelevant objects outside the current focus of attention, regardless of search mode. Physical salience appears to be a necessary condition for the activation of this type of distractor suppression, as no $\mathrm{P}_{\mathrm{D}}$ components were found in Experiments 1 and 2 for target-matching objects on the irrelevant side of heterogeneous search displays. It is notable that $\mathrm{P}_{\mathrm{D}}$ components consistently emerged later than the N2pc components in the present study, from about $280 \mathrm{~ms}$ post-stimulus (analogous to previous findings by Seiss et al., 2009), suggesting that distractor suppression was slower than the allocation of attention to target-colour objects at relevant locations. This delay may be a result of the fact that the salient distractors that were subject to inhibition always appeared in the unattended visual field, and may therefore have been detected more slowly than target-colour objects on the attended side.

The central result of the current study was that spatial filtering did not only operate during singleton search, but was also remarkably efficient when visual search was featurebased. There was some evidence in Experiment 3 that filtering mechanisms operate more effectively when observers search for singleton targets than when they search for feature-defined target objects. However, all four experiments reported here showed clear and consistent spatial filtering effects in feature-based search tasks. We also identified several factors that modulate the effectiveness of these filtering mechanisms. Non-salient target-colour objects on the irrelevant side of heterogeneous search displays generally failed to trigger N2pcs (apart from a small but reliable N2pc to such objects in the One-Colour task of Experiment 2), suggesting that spatial filtering was highly effective in preventing any capture of attention. In contrast, feature-defined singleton targets at irrelevant locations triggered reliable N2pc components in Experiments 3 and 4 , which indicates that filtering processes activated during feature-based search cannot completely prevent the occasional allocation of attention to salient objects outside the currently relevant area. In addition, Experiment 4 demonstrated that the inclusion of trials where no spatial filtering was required resulted in an additional reliable decrease in filtering efficiency on other trials. Under these conditions, spatial filtering mechanisms appear to be only partially effective when search is feature-based. The presence of attenuated but reliable $\mathrm{N} 2 \mathrm{pc}$ components to colour singletons at unattended locations is not entirely in line with the evidence for highly effective spatial filtering reported by Yantis and Jonides (1990), who found no behavioural capture effects by abrupt onset stimuli at task-irrelevant uncued locations. It is possible that the N2pc is generally more sensitive to residual attentional capture than behavioural performance measures. However, these two studies also differed with respect to the task-relevance of the singleton-defining dimension. In the experiments by Yantis and Jonides (1990), targets were shape-defined (i.e., the letters $\mathrm{E}$ or $\mathrm{H}$ ), while the onset status of objects (abrupt vs. gradual) was irrelevant for target selection. In contrast, the singleton dimension (colour) was also the target-defining dimension in the present study, and this may have reduced filtering efficiency in some conditions, resulting in some residual attentional capture by singletons at uncued locations.

How can the conclusion that feature-guided visual search is controlled by spatial filtering be reconciled with the widely shared view that feature-based attention is spatially global - a claim not only made by other authors, but also by ourselves in a recent article (Berggren \& Eimer, 2018)? First and foremost, it is important to note that in many studies of feature-based attention, its spatially global nature was inferred from the presence of reliable feature-selective modulations of visual processing outside currently relevant regions of visual space (e.g. Leblanc et al., 2008; Serences \& Boynton, 2007; Zhang \& Luck, 2009). While such findings show that spatial filtering cannot completely prevent feature-based attention from affecting the processing of stimuli at task-irrelevant locations, they do not imply that filtering is completely absent. In fact, several studies have shown that effects of feature-based attention are more pronounced at relevant as compared to irrelevant locations (e.g. Andersen et al., 2011; Martinez-Trujillo \& Treue, 2004; Saenz et al., 2002), and stronger when objects with target-matching features appear in closer proximity to taskrelevant locations (Leonard et al., 2015). These observations 
show that although spatial filtering may not be able to abolish feature-based attentional capture entirely, it can at least modulate the degree to which objects with target-matching features attract attention. This is consistent with our current finding that under certain conditions (i.e., when irrelevant objects with target-matching features are salient singletons), spatial filtering only attenuates but does not completely eliminate $\mathrm{N} 2 \mathrm{pc}$ components to these objects.

However, the feature-based attentional modulations of visual processing at irrelevant locations reported previously were typically found in tasks where objects at these locations were not particularly salient. In contrast, the N2pc results from Experiments 1 and 2 suggest that spatial filtering effectively prevents any allocation of attention to non-salient target-colour objects in heterogeneous search displays. How can this apparent discrepancy be explained? To answer this question, it is important to note that most previous experiments demonstrating the spatially global nature of feature-based attention employed procedures that were quite similar to each other, but different from typical visual search tasks. In these experiments, participants continuously monitored a stream of visual events at a relevant location (e.g. superimposed arrays of moving coloured dots, a central RSVP stream, or periodic flickering stimuli), while ignoring other visual events of the same type at irrelevant locations. Importantly, spatial attention was focused on the same region of visual space for an extended period, and the maintenance of this constant attentional focus was sustained by the continuous change of visual stimulation at this location. Most of these elements are absent in visual search tasks. Here, displays are presented as a single frame, and the positions of target and distractor objects change unpredictably across trials, so that attention has to be allocated and re-allocated to different locations within short periods of time. If spatial filtering processes are under top-down control, it is reasonable to assume that such processes are only activated when they are adaptive in facilitating task performance, by preventing interference from objects at currently irrelevant locations. Under conditions where attention is focused in a sustained fashion on a stream of rapidly changing visual events, objects at other locations are highly unlikely to attract attention away from its current locations. Because the possibility of interference is minimal in such tasks, spatial filtering may simply not be required to ensure smooth task performance. In contrast, the transient guidance of attention during feature-based visual search benefits from advance information about the location of an upcoming target object on individual trials, in particular when bottom-up salience signals are absent. When such information is available, spatial filtering mechanisms are activated at the start of each trial because they can restrict search space to currently task-relevant locations (see also Grubert \& Eimer, 2018, for evidence that featurespecific attentional templates are transiently activated and deactivated across successive trials, even when target-defining features remain constant). More generally, if the presence versus absence of spatial filtering depends on their utility for a particular selection task, as suggested here, evidence for the spatially global nature of feature-based attention obtained in one specific task setting (e.g. continuous monitoring of visual events at a fixed location) should not be generalized to also apply to a very different type of task (e.g. visual search). Feature-based attention may operate in a more global fashion under some conditions, and be subject to strong spatial filtering in other contexts.

Initial evidence that spatial filtering is most effective when it operates in a transient fashion comes from a recent visual working memory study (Allon \& Luria, 2017). Performance costs associated with the presence of distractor objects in memory sample displays were reduced when the location of these distractors was cued on a trial-by-trial basis, but not when distractor locations remained constant. These results suggest that spatial filtering settings are activated transiently, and are not maintained for extended periods of time. This might also explain why target-matching stimuli that are presented prior to search displays with feature-defined targets trigger N2pc component indicative of feature-based attentional capture, even when they appear at task-irrelevant spatial locations (Berggren \& Eimer, 2018; see also Grubert \& Eimer, 2018). On the other hand, recent work by Wang and Theeuwes (2018) has shown that interference from salient task-irrelevant distractors is reduced when these distractors appear at partially predictable locations, suggesting a link between spatial filtering and the longer-term acquisition of knowledge about statistical regularities. Thus, the question of whether and when filtering needs to be activated transiently or in a more sustained fashion in order to be effective clearly requires further investigation. Another important question concerns limitations in the flexibility of spatial filtering during feature-based search. For example, previous work (Berggren et al., 2017) has shown that participants are unable to assign particular feature values to specific spatial regions (e.g. "attend to red items on the left and blue items on the right"). Here, target-colour objects elicited equivalent attentional biases irrespective of whether they appeared at their respective task-relevant locations or on the opposite side, suggesting that the division of visual space into relevant and irrelevant regions by spatial filtering cannot operate in a feature-contingent fashion.

In summary, the present study demonstrates that spatial filtering processes control the allocation of attention both during singleton and feature-based visual search. Our results confirm previous suggestions that salient distractors outside the current attentional window do not capture attention in singleton search tasks, and suggest that these distractors may be actively suppressed. Critically, the finding that spatial filtering mechanisms strongly affect feature-guided attentional selection processes has important implications for the prevalent view that feature-based attention operates in a spatially global 
fashion. We propose that the effects of feature-based attention can be transiently restricted to relevant locations when this is adaptive for task performance, as is the case in feature-guided visual search tasks.

Acknowledgements This work was supported by grant ES/L016400/1 from the Economic and Social Research Council (ESRC), UK. Author NB is also supported by grant ES/R003459/1 from the ESRC.

Open Practices Statement Data and materials for all experiments are available from the authors upon request. None of the experiments were preregistered.

\section{References}

Allon, A.S., \& Luria, R. (2017). Compensation mechanisms that improve distractor filtering are short-lived. Cognition, 164, 74-86.

Andersen, S.K., Fuchs, S., \& Müller, M.M. (2011). Effects of featureselective and spatial attention at different stages of visual processing. Journal of Cognitive Neuroscience, 23, 238-246.

Andersen, S.K., Hillyard, S.A., \& Müller, M.M. (2013). Global facilitation of attended features is obligatory and restricts divided attention. Journal of Neuroscience, 33, 18200-18207.

Bacon, W.F., \& Egeth, H.E. (1991). Local processes in preattentive feature detection. Journal of Experimental Psychology: Human Perception and Performance, 17, 77-90.

Bacon, W.F., \& Egeth, H.E. (1994). Overriding stimulus-driven attentional capture. Perception \& Psychophysics, 55, 485-496.

Barras, C., \& Kerzel, D. (2016). Active suppression of salient-butirrelevant stimuli does not underlie resistance to visual interference. Biological Psychology, 121, 74-83.

Barras, C., \& Kerzel, D. (2017). Salient-but-irrelevant stimuli cause attentional capture in difficult, but attentional suppression in easy visual search. Psychophysiology, 54, 1826-1838.

Belopolsky, A.V., \& Theeuwes, J. (2010). No capture outside the attentional window. Vision Research, 50, 2543-2550.

Belopolsky, A.V., Zwaan, L., Theeuwes, J., \& Kramer, A.F. (2007). The size of an attentional window modulates attentional capture by color singletons. Psychonomic Bulletin \& Review, 14, 934-938.

Berggren, N., \& Eimer, M. (2018). Feature-guided attentional capture cannot be prevented by spatial filtering. Biological Psychology, 134, 1-8.

Berggren, N., Jenkins, M., McCants, C.W., \& Eimer, M. (2017). The spatially global control of attentional target selection in visual search. Visual Cognition, 25, 196-214.

Bravo, M.J., \& Nakayama, K. (1992). The role of attention in different visual-search tasks. Perception \& Psychophysics, 51, 465-472.

Brignani, D., Lepsien, J., \& Nobre, A.C. (2010). Purely endogenous capture of attention by task-defining features proceeds independently from spatial attention. NeuroImage, 51, 859-866.

Burnham, B.R. (2018). Selectively ignoring locations does not modulate contingent involuntary orienting, but selectively attending does. Visual Cognition, 26, 48-70.

Callahan-Flintoft, C., \& Wyble, B. (2017). Non-singleton colors are not attended faster than categories, but they are encoded faster: A combined approach of behavior, modelling and ERPs. Vision Research, 140, 106-119.

Duncan, J., \& Humphreys, G. (1992). Beyond the search surface: Visual search and attentional engagement. Journal of Experimental Psychology: Human Perception and Performance, 18, 578-588.
Eimer, M. (1996). The N2pc component as an indicator of attentional selectivity. Electroencephalography and Clinical Neurophysiology, 99, 225-234.

Eimer, M., \& Kiss, M. (2008). Involuntary Attentional Capture is Determined by Task Set: Evidence from Event-related Brain Potentials. Journal of Cognitive Neuroscience, 20, 1423-1433.

Eimer, M., \& Kiss, M. (2010). Top-down search strategies determine attentional capture in visual search: Behavioral and electrophysiological evidence. Attention, Perception, \& Psychophysics, 72, 951962.

Feldmann-Wüstefeld, T., \& Schubö, A. (2015). Target discrimination delays attentional benefit for grouped contexts: An ERP study. Brain Research, 1629, 196-209.

Folk, C.L., Leber, A.B., \& Egeth, H.E. (2002). Made you blink! Contingent attentional capture produces a spatial blink. Perception \& Psychophysics, 64, 741-753.

Folk, C.L., Remington, R.W., \& Johnston, (1992). Involuntary covert orienting is contingent on attentional control settings. Journal of Experimental Psychology: Human Perception and Performance, $24,847-858$

Fortier-Gauthier, U., Moffat, N., Dell'Acqua, R., McDonald, J.J., \& Jolicoeur, P. (2012). Contralateral cortical organisation of information in visual short-term memory: Evidence from lateralized brain activity during retrieval. Neuropsychologia, 50, 1748-1758.

Gaspelin, N., \& Luck, S.J. (2018). The role of inhibition in avoiding distraction by salient stimuli. Trends in Cognitive Sciences, 22, 7992.

Grubert, A., \& Eimer, M. (2015). Rapid parallel target selection in singlecolor and multiple-color visual search. Journal of Experimental Psychology: Human Perception and Performance, 41, 86-101.

Grubert, A., \& Eimer, M. (2018). The time course of target template activation processes during preparation for visual search. Journal of Neuroscience, 38, 9527-9538.

Hickey, C., Di Lollo, V., \& McDonald, J.J. (2009). Electrophysiological indices of target and distractor processing in visual search. Journal of Cogntiive Neuroscience, 21, 760-775.

Kerzel, D., Born, S., \& Schönhammer, J. (2012). Perceptual grouping allows for attention to cover non-contiguous locations and suppress capture from nearby locations. Journal of Experimental Psychology: Human Perception and Performance, 38, 1362-1370.

Leblanc, É., Prime, D., \& Jolicoeur, P. (2008). Tracking the Location of Visuospatial Attention in a Contingent Capture Paradigm. Journal of Cognitive Neuroscience, 20, 657-671.

Leonard, C.J., Balestreri, A., \& Luck, S.J. (2015). Interactions between space-based and feature-based attention. Journal of Experimental Psychology: Human Perception and Performance, 41, 11-16.

Luck, S.J., \& Hillyard, S.A. (1994). Spatial filtering during visual search: Evidence from human electrophysiology. Journal of Experimental Psychology: Human Perception and Performance, 20, 1000-1014.

Martinez-Trujillo, J.C., \& Treue, S. (2004). Feature-based attention increases the selectivity of population responses in primate visual cortex. Current Biology, 14, 744-751.

Mazza, V., Turatto, M., \& Caramazza, A. (2009). Attention selection, distractor suppression and N2pc. Cortex, 45, 879-890.

Mazza, V., Turatto, M., Umiltà, C., \& Eimer, M. (2007). Attentional selection and identification of visual objects are reflected by distinct electrophysiological responses. Experimental Brain Research, 181, 531-536.

Posner, M.I. (1980). Orienting of attention. Quarterly Journal of Experimental Psychology, 32, 3-25.

Ruthruff, E., \& Gaspelin, N. (2018). Immunity to attentional capture at ignored locations. Attention, Perception, \& Psychophysics, 80, 325 336.

Saenz, M., Buracas, G.T., \& Boynton, G.M. (2002). Global effects of feature-based attention in human visual cortex. Nature Neuroscience, 5, 631-632. 
Sawaki, R., Geng, J.J., \& Luck, S.J. (2012). A common neural mechanism for preventing and terminating the allocation of attention. Journal of Neuroscience, 32, 10725-10736.

Sawaki, R., \& Luck, S.J. (2010). Capture versus suppression of attention by salient singletons: Electrophysiological evidence for an automatic attend-to-me signal. Attention Perception \& Psychophysics, 72, $1455-1470$.

Seiss, E., Kiss, M., \& Eimer, M. (2009). Does focused endogenous attention prevent attentional capture in pop-out visual search? Psychophysiology, 46, 703-717.

Serences, J.T., \& Boynton, G.M. (2007). Feature-based attentional modulations in the absence of direct visual stimulation. Neuron, 55, 301312.

Stothart, C., Simons, D.J., Boot, W.R., \& Wright, T.J. (2019). What to where: The right attention set for the wrong location. Perception, 48, 602-615.

Theeuwes, J. (2010). Top-down and bottom-up control of visual selection. Acta Psychologica, 135, 77-99.

Theeuwes, J., Kramer, A.F., \& Atchley, P. (2001). Spatial attention in early vision. Acta Psychologica, 108, 1-20.
Vatterott, D.B., \& Vecera, S.P. (2015). The attentional window configures to object and surface boundaries. Visual Cognition, 23, 561-576.

Wang, B., \& Theeuwes, J. (2018). Statistical regularities modulate attentional capture. Journal of Experimental Psychology: Human Perception and Performance, 44, 13-17.

Wolfe, J.M., \& Horowitz, T.S. (2004). What attributes guide the deployment of visual attention and how do they do it? Nature Reviews Neuroscience, 5, 495-501.

Yantis, S., \& Jonides, J. (1984). Abrupt visual onsets and selective attention: Evidence from visual search. Journal of Experimental Psychology: Human Perception and Performance, 10, 601-621.

Yantis, S., \& Jonides, J. (1990). Abrupt visual onsets and selective attention: Voluntary versus automatic allocation. Journal of Experimental Psychology: Human Perception and Performance, 16, 121-134.

Zhang, W., \& Luck, S.J. (2009). Feature-based attention modulates feedforward visual processing. Nature Neuroscience, 12, 24-25.

Publisher's note Springer Nature remains neutral with regard to jurisdictional claims in published maps and institutional affiliations. 